\title{
Reflection, Transmission, and Refraction
}

\section{Contents}

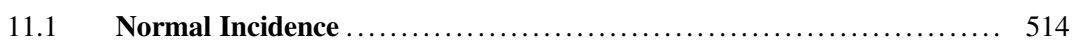

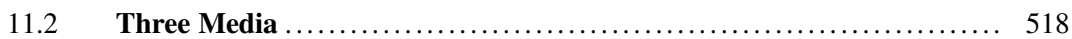

11.2.1 A Limp Diaphragm Separating Two Gases ....................... 520

11.2.2 An Impedance Matching Antireflective Layer ..................... 520

11.2.3 The "Mass Law" for Sound Transmission Through Walls .............. 520

11.2.4 Duct Constriction/Expansion Low-Pass Filters ..................... 521

11.3 Snell's Law and Fermat's Principle ............................. 523

11.3.1 Total Internal Reflection .................................... 526

11.3.2 The Rayleigh Reflection Coefficient ........................... 528

$11.4 \quad$ Constant Sound Speed Gradients .............................. 529

11.4.1 Constant Gradient's Equivalence to Solid Body Rotation ................ 531

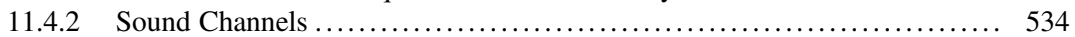

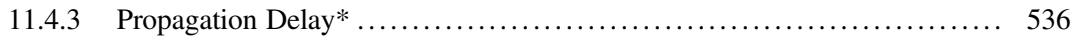

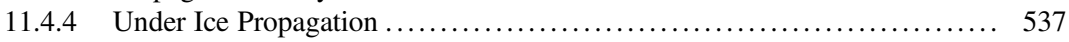

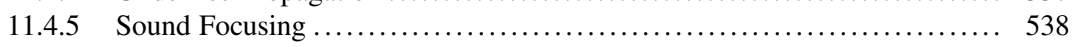

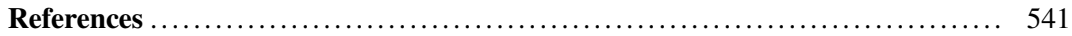

In Chap. 10, we transitioned from a lumped-element perspective to a formalism which treated fluids as continuous media that include the effects of both compressibility and inertia throughout. We described disturbances from equilibrium using a wave equation and focused attention on planewaves that could be described as propagating along one spatial dimension. In this chapter, we will examine the behavior of such one-dimensional waves propagating through media that are not homogeneous. We start with an examination of the behavior of planewaves impinging on a planar interface between two fluid media with different properties and then extend that analysis to multiple interfaces and to waves that impinge on the interface from an angle that is not perpendicular to that surface. This exploration concludes with consideration of wave propagation through a medium whose properties change slowly and continuously through space.

What does "slowly" mean in the previous sentence? To answer that question, we will break down the general problem of propagation through an inhomogeneous medium into two limiting cases. As before, such limiting cases suggest appropriate (usually simple) analytical approaches that will develop useful intuition for understanding cases that may be intermediate between the simpler limits. In this 
chapter, the interesting limits depend on the scale of the medium's inhomogeneity relative to the wavelength of the sound that impinges on it (from afar, since we consider only planewaves). If a wave encounters a deviate region with a size that is on the order of its own wavelength or smaller, and that region has a different density, a different compressibility, or both, then we treat the inhomogeneous region as a "scatterer." Those cases will be examined in Chap. 12 when the problem of radiation in three dimensions is analyzed and the scattering body will be driven by the impinging sound wave causing the ensonified region to behave as a radiating "source." That "scattered" sound field will be superimposed on the incident sound field.

In this chapter, we will consider the opposite limit, where the size of the boundary that separates regions with different acoustical properties is much larger than the wavelength of the sound. In fact, many cases to be examined here will assume that the extent of the boundary is infinite. In those cases, the problem is treated as one where the wave incident on such an interface will be both reflected back into the medium from which it originated and be transmitted into the second medium on the other side of the interface.

For the case where the interface is not discontinuous, what we will mean by requiring that the medium's properties (e.g., sound speed and density) "are varying slowly" will again be related to the rate at which the property changes in space relative to the wavelength of the sound. For example, if we specify the change in sound speed with position, $d c / d z \equiv g$, then $g$ will have the units $\mathrm{m} / \mathrm{s}$ per $\mathrm{m}$, which is equivalent to a frequency. The wave also has a characteristic frequency, $f=c / \lambda$, that is the ratio of the sound speed to the wavelength. If $g \ll f$, then any significant change in sound speed will occur over a very large number of wavelengths.

\subsection{Normal Incidence}

All of us are familiar with echoes, whether produced by a handclap reflected from a large building; a loud "hello" shouted from a precipice and reflected from a bluff, as diagrammed in Fig. 11.1; or a "flutter echo" produced by some impulsive sound source reverberating between long parallel walls. In those cases, a sound wave in air impinges on a rigid solid surface. The air's particle velocity that accompanies the pressure wave (via the Euler equation) cannot penetrate the solid. To satisfy this rigid boundary condition, we can imagine a sound wave of equal amplitude, but propagating in the opposite direction, coming from within the solid toward the interface, just as was done when examining the reflection of a pulse propagating along a string in Sect. 3.2. As justified in Sect. 12.4.1, the superposition of the two waves cancels the velocity at the interface, satisfying the condition that the interface is impenetrable to the gas, while doubling the acoustic pressure amplitude on that surface.

To compare how a real wave reflects in the "rigid boundary" case (and to determine how "rigidly" the boundary behaves), let's say the magnitude of the incident planewave, $\left|\widehat{\mathbf{p}}_{\mathbf{i}}\right|$, approaching the boundary is $94 \mathrm{~dB}_{\mathrm{SPL}} \Rightarrow\left|\widehat{\mathbf{p}}_{\mathbf{i}}\right|=\sqrt{2} \mathrm{~Pa}=1.0 \mathrm{~Pa}_{r m s}$. Again, we focus on a single-frequency wave and indicate the incident wave's amplitude and phase by the complex phasor, $\widehat{\mathbf{p}}_{i}$. The time-averaged intensity of a $94 \mathrm{~dB}_{\mathrm{SPL}}$ planewave in air is $\left\langle I_{\text {air }}\right\rangle_{t}=\left|\widehat{\mathbf{p}}_{\mathbf{i}}\right|^{2} /(2 \rho c)_{\text {air }}=10^{(94 / 10)} \times 10^{-12} \mathrm{~W} / \mathrm{m}^{2} \cong$ $2.5 \mathrm{~mW} / \mathrm{m}^{2}$. (If this is not instantaneously obvious, you need to review Sect. 10.5.1.)

Let's also say the wave was reflected from a concrete wall. Since the pressure amplitude at the wall is double that of the sound wave far from the wall, and that the pressure is continuous across the interface, we can calculate the intensity of the sound that entered the wall. The density of concrete is about $2600 \mathrm{~kg} / \mathrm{m}^{3}$ and the speed of compressional waves in concrete is about $3100 \mathrm{~m} / \mathrm{s}$, so $(\rho c)_{\text {concrete }}=8.06 \mathrm{MPa}-\mathrm{s} / \mathrm{m}$. Since the pressure at the wall is twice that of the wave in air, $\left|\widehat{\mathbf{p}}_{\mathbf{t}}\right|=$ 

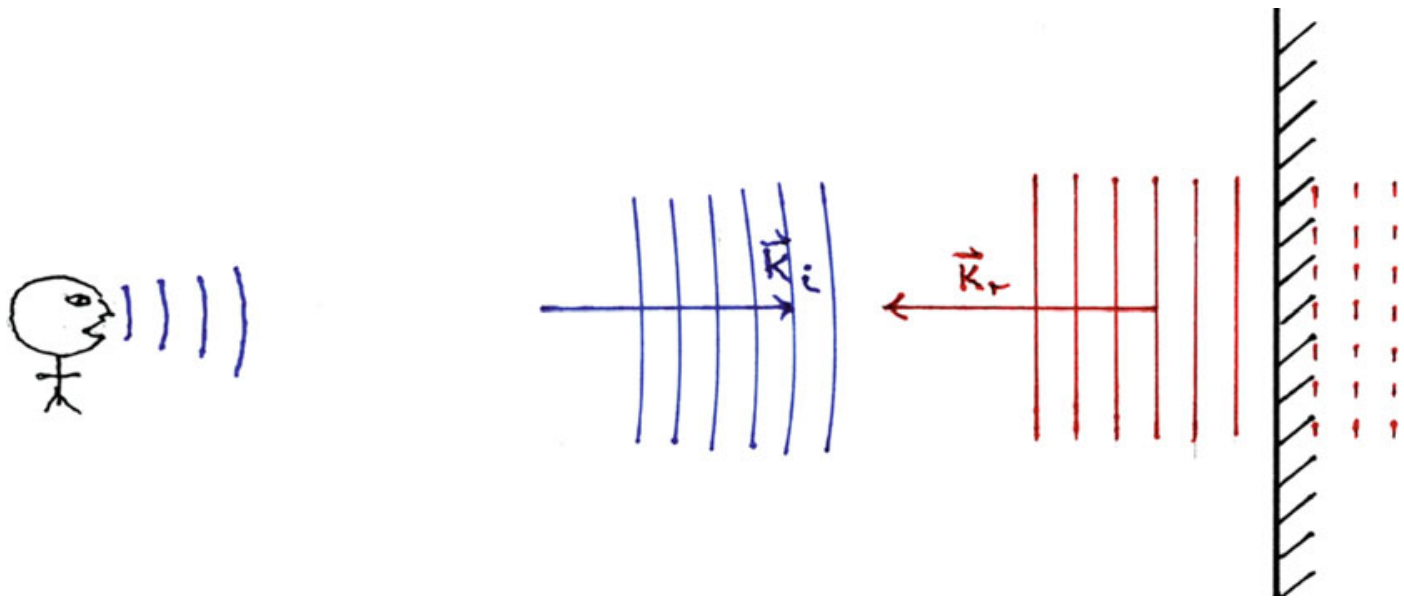

Fig. 11.1 The sound produced by this person is nearly a planewave (wave fronts shown in blue), characterized by the wavenumber, $\vec{k}_{i}$, when it is reflected by a large flat rigid surface. The duration of the sound pulse is sufficiently short that the reflected wave, characterized by $\vec{k}_{r}$, and the incident wave only interact near the surface. To satisfy the condition that no fluid can enter or leave the solid interface, we imagine another planewave of equal amplitude traveling back (with wave fronts shown in red) toward the source. Where the incident and reflected wave superimpose at the rigid surface, the pressure is doubled, but the slope of the pressure, $(d p / d x)_{x=0}$, evaluated at the interface, is zero. By Euler's equation, the total acoustic particle velocity normal to the surface vanishes, as required

$2\left|\widehat{\mathbf{p}}_{\mathbf{i}}\right|=2 \sqrt{2} \mathrm{~Pa}$, the time-averaged intensity of the sound penetrating the concrete is $\left\langle I_{\text {concrete }}\right\rangle_{t}=$ $\left|\widehat{\mathbf{p}}_{\mathbf{t}}\right|^{2} /(2 \rho c)_{\text {concrete }}=0.5 \mu \mathrm{W} / \mathrm{m}^{2}$, less than $0.02 \%$ of the intensity of the sound in air.

The equations developed in this chapter can be used to show that the amplitude of a wave in air that is reflected from a concrete wall is nearly the same as the incident wave, but not quite. Our assumption that the echo had the same amplitude as the incident sound wave was just as solid as the concrete.

We can also compare the particle velocity of the sound in air with that of the compressional wave within the concrete. In dry air, a $94 \mathrm{~dB}_{\mathrm{SPL}}$ sound wave would have a particle velocity amplitude of $\left|\widehat{\mathbf{v}}_{\mathbf{i}}\right|=\left|\widehat{\mathbf{p}}_{\mathbf{i}}\right| /(\rho c)_{\text {air }}=3.3 \mathrm{~mm} / \mathrm{s}$. In the concrete, the pressure of the transmitted wave is twice that in air (far from the wall), but $\left|\widehat{\mathbf{v}}_{\mathbf{t}}\right|=2\left|\widehat{\mathbf{p}}_{\mathbf{i}}\right| /(\rho c)_{\text {concrete }}=0.35 \mu \mathrm{m} / \mathrm{s}$. Again, we see that our assumption of a rigid and immobile boundary was good to about one part in ten thousand $(0.01 \%)$. We can check our intensity results using Eq. (10.36), since we also know $\langle I\rangle_{t}=\left|\widehat{\mathbf{p}}_{\mathbf{t}}\right|\left|\widehat{\mathbf{v}}_{\mathbf{t}}\right| / 2$, since $\widehat{\mathbf{p}}_{\mathbf{t}}$ and $\widehat{\mathbf{v}}_{\mathbf{t}}$ are in-phase for our assumed traveling wave. In dry air at one atmosphere, $p_{m}=101,325 \mathrm{~Pa}$, and letting $T_{m}=7{ }^{\circ} \mathrm{C}$, $(\rho c)_{\text {air }}=423 \mathrm{~Pa}-\mathrm{s} / \mathrm{m}$. This gives $\left\langle I_{\text {air }}\right\rangle_{t}=2.4 \mathrm{~mW} / \mathrm{m}^{2}$ and in the concrete $\left\langle I_{\text {concrete }}\right\rangle_{t}=0.5 \mu \mathrm{W} / \mathrm{m}^{2}$, in good agreement with the earlier calculation, as must be the case.

Using Eq. (9.38), the power dissipated per unit area due to the thermal relaxation losses at the interface can be calculated and compared to the incident intensity, since the concrete wall will behave as an isothermal boundary. ${ }^{1}$ Since this loss mechanism is frequency dependent, let's do the calculation for $f=1 \mathrm{kHz}$. From the DeltaEC Thermophysical Properties of air at $300 \mathrm{~K}$ and $1 \mathrm{bar}=10^{5} \mathrm{~Pa}$,

\footnotetext{
${ }^{1}$ To determine if the concrete wall forces the air that it contacts to behave isothermally, we can calculate the heat capacity per unit area that is contained within a layer of the material that is one thermal penetration depth thick, $\rho c_{P} \delta_{\kappa}$. Using the thermophysical properties available in DeltaEC, $\left(\rho c_{P} \delta_{\kappa}\right)_{\text {air }}=0.10 \mathrm{~J} / \mathrm{m}^{2}-{ }^{\circ} \mathrm{C}$. Approximate values for concrete are $\rho=2600 \mathrm{~kg} / \mathrm{m}^{3}, c_{P}=880 \mathrm{~J} / \mathrm{kg}^{\circ}{ }^{\circ} \mathrm{C}$, and $\kappa=0.29 \mathrm{~W} / \mathrm{m}^{-}{ }^{\circ} \mathrm{C}$, so at $1 \mathrm{kHz}, \delta_{\kappa}=6.35 \mu \mathrm{m}$ and $\left(\rho c_{P} \delta_{\kappa}\right)_{\text {Concrete }}=14.5 \mathrm{~J} /$ $\mathrm{m}^{2}{ }^{\circ} \mathrm{C} \gg\left(\rho c_{P} \delta_{\kappa}\right)_{\text {air }}=0.10 \mathrm{~J} / \mathrm{m}^{2}-{ }^{\circ} \mathrm{C}$. For a more detailed discussion, see Eq. (59) for $\varepsilon_{s}$ in G. W. Swift, "Thermoacoustic engines,” J. Acoust. Soc. Am. 84(4), 1145-1180 (1988).
} 


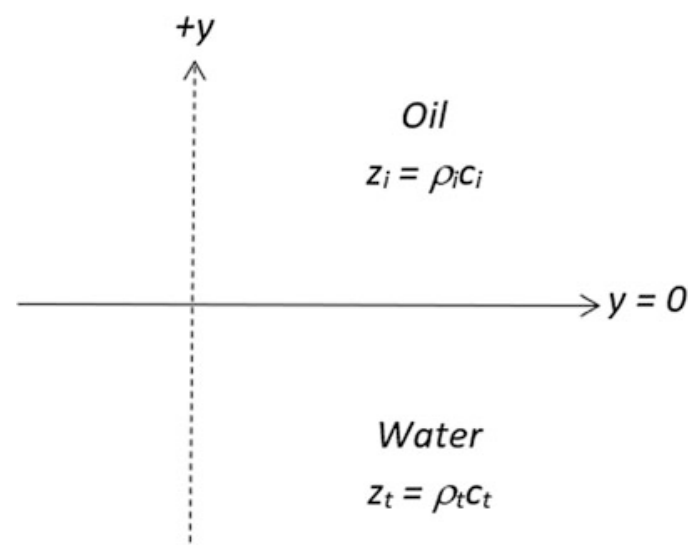

Fig. 11.2 Coordinate system for two fluids that can be thought of as oil floating on water. The dashed line is normal to the plane interface between the two fluids at $y=0$. In this diagram, it is assumed that the sound originates from the oil which has a specific acoustic impedance of $z_{i}=\rho_{i} c_{i}$. A wave will be transmitted across the interface into a second medium (water) with a specific acoustic impedance $z_{t}=\rho_{t} c_{t}$

$\delta_{\kappa}=84.6 \mu \mathrm{m}$, corresponding to a thermal absorption of $0.76 \mu \mathrm{W} / \mathrm{m}^{2}$. This is a miniscule loss $(0.03 \%)$, although thermal relaxation at the interface is responsible for a slightly larger decrease in the reflected amplitude, at this frequency, than the intensity of the sound that enters the concrete. Why do we not need to calculate the viscous boundary layer dissipation?

Now that we have the behavior of a common reflection situation "under our belts," we can start our formal investigation of reflection and transmission of planewaves impinging normally on an interface between any two dissimilar media. For these calculations, keep in mind a very simple case of two immiscible liquids in a uniform gravitational field. Let's assume that we have a sea of oil floating on a sea of water, as shown in Fig. 11.2, with the "up" direction being $+y$ and $y=0$ at the interface between the two liquids. The mass density of the oil, $\rho_{\text {oil }}$, is less than the density of water. To simplify the specification of variables on either side of the interface, we will designate parameters of the wave and of the medium above the interface $(y>0)$ with the subscript, $i$ (incident), and those below the interface $(y<0)$ with the subscript, $t$ (transmitted).

Let's imagine that a single-frequency plane sound wave originates far above the interface and is propagating in the $-y$ direction. That wave excites the interface with a pressure amplitude, $\left|\widehat{\mathbf{p}}_{\mathbf{i}}\right|$. We can express the pressure amplitude as a function of $y$ and $t$ above the interface

$$
p_{i}(y, t)=\mathfrak{R} e\left[\widehat{\mathbf{p}}_{\mathbf{i}} e^{j\left(\omega t+k_{i} y\right)}\right] \quad \text { for } \quad y>0 .
$$

That incident plane pressure wave also has an associated fluid particle velocity, $\widehat{\mathbf{v}}_{\mathbf{i}}$, that is related to the pressure by the Euler equation.

$$
v_{i}(y, t)=\mathfrak{R} e\left[\frac{\widehat{\mathbf{p}}_{\mathbf{i}}}{\rho_{i} c_{i}} e^{j\left(\omega t+k_{i} y\right)}\right]=\mathfrak{R} e\left[\frac{\widehat{\mathbf{p}}_{\mathbf{i}}}{z_{i}} e^{j\left(\omega t+k_{i} y\right)}\right] \quad \text { for } \quad y>0
$$

In the right-hand term of Eq. (11.2), $z_{i}$ is the specific acoustic impedance of the oil. Using our generic expression, Eq. (10.21), for the sound speed, $c$, in a fluid with an adiabatic bulk modulus, $B_{s}, c \equiv$ $\sqrt{B_{s} / \rho}$, we see that the specific acoustic impedance is a combination of the fluid's density and compressibility, $z=\rho c=\sqrt{\rho B_{s}}$. 
As for the echo case, there will be a wave that is reflected at the interface that will travel in a direction opposite to that of the incident wave.

$$
p_{r}(y, t)=\mathfrak{R} e\left[\widehat{\mathbf{p}}_{\mathbf{r}} e^{j\left(\omega t-k_{i} y\right)}\right] \text { for } \quad y>0
$$

The presence of the incident wave will excite motion in that interface which will also generate a wave propagating into the water as well as the wave that is reflected back into the oil. To demonstrate the necessity for the simultaneous existence of the incident, reflected, and transmitted waves, we need to consider the properties of the interface between the two media.

Since we will treat these media and the waves they contain as a linear system, we can guarantee that the reflected and transmitted waves both have the same frequency as the incident wave: $\omega=\omega_{i}=\omega_{r}=\omega_{t}$. As we have seen for the simple harmonic oscillator, at steady state, the forced linear system can only respond at the forcing frequency. It is the incident wave that is forcing the motion of the interface. Since the frequencies of all of the waves must be the same, and the sound speeds in the two media might differ, the wavelength of the sound in the water will be different than that in the oil: $\lambda_{t}=c_{t} / f=2 \pi / k_{t}$.

$$
p_{t}(y, t)=\mathfrak{R} e\left[\widehat{\mathbf{p}}_{\mathbf{t}} e^{j\left(\omega t+k_{t} y\right)}\right] \text { for } y<0
$$

Our second requirement at the interface is that the two fluids always remain in contact. There are cases where the amplitude of the incident wave is sufficiently large to produce a vapor cavity at the interface, but we will limit our attention here to wave amplitudes that are insufficient to create such cavitation effects [1]. In most practical cases, the boundaries do not separate, so the normal velocities of the two fluids must match at the interface for all times.

$$
v_{i}(0, t)+v_{r}(0, t)=v_{t}(0, t)
$$

Our final requirement is dictated by the fact that the interface, $y=0$, is only a mathematical construct - it has no mass. Since that interface is massless, Newton's Second Law of Motion would guarantee that any pressure difference across the interface would produce a non-zero force that would create infinite fluid accelerations. To guarantee that does not happen, we require that the pressure be continuous across that interface for all time.

$$
p_{i}(0, t)+p_{r}(0, t)=p_{t}(0, t)
$$

Those three conditions are sufficient to completely determine the behavior of the waves at the interface when we recognize that $v_{t}(y, t)$ is related to $p_{t}(y, t)$ by the specific acoustic impedance of the water, $z_{t}$. Taking the ratio of Eq. (11.6) to Eq. (11.5), the amplitude ratio of the reflected and transmitted waves to the amplitude of the incident wave can be calculated.

$$
\frac{p_{i}(0, t)+p_{r}(0, t)}{v_{i}(0, t)+v_{r}(0, t)}=\frac{p_{t}(0, t)}{v_{t}(0, t)}=z_{t}
$$

Recognizing that the incident and reflected waves are both in the oil, but are traveling in opposite directions, the magnitude of the specific acoustic impedance, $\left|z_{i}\right|$, is the same for both waves, but their signs are opposite. This leads to the further simplification of Eq. (11.7).

$$
z_{i} \frac{p_{i}(0, t)+p_{r}(0, t)}{p_{i}(0, t)-p_{r}(0, t)}=-z_{t}
$$


Again, because we are limiting ourselves to linear acoustics, it is only the ratios of the amplitudes that have any significance. If we double the amplitude of the incident wave, the amplitudes of the reflected and transmitted waves must also double. It is therefore reasonable to define a pressure reflection coefficient, $\mathbf{R} \equiv \widehat{\mathbf{p}}_{\mathbf{r}} / \widehat{\mathbf{p}}_{\mathbf{i}}$, as we did in Eq. (10.105). With that definition and a little algebraic manipulation, Eq. (11.8) can be re-written in a compact form to represent the magnitude of the pressure reflection coefficient, $R$.

$$
R \equiv \frac{\widehat{\mathbf{p}}_{\mathbf{r}}}{\widehat{\mathbf{p}}_{\mathbf{i}}}=\frac{z_{t}-z_{i}}{z_{t}+z_{i}}=\frac{\left(z / z_{i}\right)-1}{\left(z_{t} / z_{i}\right)+1}
$$

This result makes sense and is reminiscent of Eq. (10.105). If the two media have the same specific acoustic impedances, there is no reflection. Plugging in the specific acoustic impedances for air and concrete produces the same result as we obtained for the "echo" example that began this treatment and produced nearly perfect reflection: $R=0.9999$.

In the case of oil over water, as shown in Fig. 11.2, then $z_{i}<z_{t}$ so $R$ is positive and the phase of the reflected wave will be the same as the incident wave, although their amplitudes will differ, similar to the in-phase pulse reflection in Fig. 3.4. If the situation were reversed so that the incident wave originated in the water, then $z_{i}>z_{t}$ so $R$ is negative and the phase of the reflected wave will be inverted, as shown for the pulses in Fig. 3.3.

Defining the pressure transmission coefficient in a similar way, $T \equiv \widehat{\mathbf{p}}_{\mathbf{t}} / \widehat{\mathbf{p}}_{\mathbf{i}}$, the pressure continuity boundary condition of Eq. (11.6) can now be written as $1+R=T$.

$$
T \equiv \frac{\widehat{\mathbf{p}}_{\mathbf{t}}}{\widehat{\mathbf{p}}_{\mathbf{i}}}=\frac{2 z_{t}}{z_{t}+z_{i}}=\frac{2\left(z / z_{i}\right)}{\left(z_{t} / z_{i}\right)+1}
$$

The factor of two is reassuring, since we expected pressure doubling at the interface in the echo example where $z_{t} \gg z_{i}$, and we saw the same factor of two in Eq. (10.105).

For oil above water, we can use $\rho_{\text {oil }}=950 \mathrm{~kg} / \mathrm{m}^{3}, c_{\text {oil }}=1540 \mathrm{~m} / \mathrm{s}, z_{\text {oil }}=(\rho c)_{\text {oil }}=1.463 \times 10^{6}$ $\mathrm{Pa}-\mathrm{s} / \mathrm{m}$, and $\rho_{\text {water }}=998 \mathrm{~kg} / \mathrm{m}^{3}, c_{\text {water }}=1481 \mathrm{~m} / \mathrm{s}$, and $z_{\text {water }}=(\rho c)_{\text {water }}=1.478 \times 10^{6} \mathrm{~Pa}-\mathrm{s} / \mathrm{m}$. The specific acoustic impedance of these two liquids is very close, so for a planewave originating in the oil, $R=0.005$ and $T=1.005$. If the planewave originated in the water, $R=-0.005$ and $T=0.995$. The fact that $R$ is negative for sound traveling from water into oil indicates that the wave reflected back into the water from the interface is reflected with a $180^{\circ}$ phase shift with respect to the incident wave.

If Fig. 11.2 represented air over water, as on a lake, then the impedance contrast would be similar to the echo example, if we assume dry air at one atmosphere, $p_{m}=101,325 \mathrm{~Pa}$, and $T_{m}=20^{\circ} \mathrm{C}$, then $(\rho c)_{\text {air }}=413 \mathrm{~Pa}-\mathrm{s} / \mathrm{m}$. If the wave originated in the air, the results would be similar to the echo example. If the wave originated in the water, $R=-0.9994$, so again the reflection is almost perfect, but the reflected wave is $180^{\circ}$ out-of-phase with incident wave. The pressure transmission coefficient is $T=0.00056$, so we call that interface, in such a situation (i.e., the wave originating in the medium of higher specific acoustic impedance), a pressure release boundary.

\subsection{Three Media}

If we had two gases in contact, then we would need a membrane to keep them from diffusing into each other. Similarly, we might be interested in the transmission of sound between two living spaces separated by a wall. There is no reason why we could not extend the same style of argument just employed with one interface between two media. We would need five waves instead of three since the 


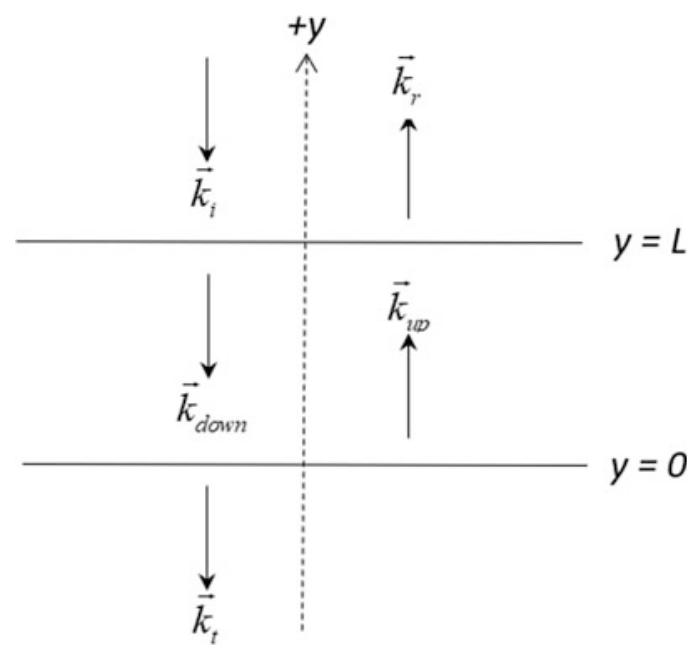

Fig. 11.3 For a planewave that propagates through three media, bounded by two parallel interfaces, two additional waves need to be included for propagation through the central region. In this diagram, two waves are "trapped" inside the central medium, between $y=0$ and $y=L$, that has a specific acoustic impedance of $z_{c}=\rho_{c} c_{c}$. The wavevector of the incident planewave, $\vec{k}_{i}$, and the reflected wavevector, $\vec{k}_{r}$, are both in the upper medium with $z_{i}=\rho_{i} c_{i}$. The transmitted wave represented by the wavevector, $\vec{k}_{t}$, is in the lower medium, with specific acoustic impedance $z_{t}=\rho_{t} c_{t}$. All wave vectors are colinear and normal to both interfaces

central region, with specific acoustic impedance, $z_{c}$, can support both waves moving up and moving down, as shown in Fig. 11.3.

$$
p_{u p}(y, t)=\mathfrak{R} e\left[\widehat{\mathbf{p}}_{\mathbf{u p}} e^{j\left(\omega t-k_{c} y\right)}\right] \quad \text { and } \quad p_{\text {down }}(y, t)=\mathfrak{R} e\left[\widehat{\mathbf{p}}_{\mathbf{d o w n}} e^{j\left(\omega t+k_{c} y\right)}\right]
$$

Again, linearity guarantees that these two new waves also have a frequency $\omega=\omega_{\text {up }}=\omega_{\text {down }}$, so their wavelengths and wavenumbers depend upon the speed of sound in the central medium: $\lambda_{c}=c_{c} / f=2 \pi /\left|\vec{k}_{c}\right|$. We would need to impose the continuity of normal particle velocity (11.5) and the continuity of pressure (11.6) on two planes, $y=0$ and $y=L$, if the central medium has thickness, $L$. We are again only interested in the amplitude ratios (i.e., specification of the incident amplitude, $\widehat{\mathbf{p}}_{\mathbf{i}}$, is still arbitrary) and can calculate the complex pressure reflection coefficient, $\mathbf{R}$.

$$
\mathbf{R}=\frac{\left(1-z_{2 t}\right) \cos \left(k_{c} L\right)+j\left(z_{c} / z_{t}-z_{i} / z_{c}\right) \sin \left(k_{c} L\right)}{\left(1+z_{i} / z_{t}\right) \cos \left(k_{c} L\right)+j\left(z_{c} / z_{t}+z_{i} / z_{c}\right) \sin \left(k_{c} L\right)}
$$

The power transmission coefficient will be a scalar, as it was in Eq. (10.109), and will be related to the reflection coefficient through energy conservation, as it was in Eq. (10.110).

$$
T_{\Pi}=\frac{4}{2+\left(z / z_{i}+z_{i} / z_{t}\right) \cos ^{2}\left(k_{c} L\right)+\left(z_{c}^{2} / z_{t} z_{i}+z_{t} z_{i} / z_{c}^{2}\right) \sin ^{2}\left(k_{c} L\right)}
$$

In many cases, the incident and transmitted media are the same, $z_{i}=z_{t}$, simplifying the power transmission coefficient.

$$
T_{\Pi}=\left[1+(1 / 4)\left(z_{c} / z_{i}-z_{i} / z_{c}\right)^{2} \sin ^{2}\left(k_{c} L\right)\right]^{-1} \quad \text { if } \quad z_{i}=z_{t}
$$




\subsubsection{A Limp Diaphragm Separating Two Gases}

If we apply Eq. (11.12) to the case of two different gases separated by a membrane with $z_{c} \gg z_{i}$ and $z_{c} \gg z_{t}$, which is so thin (i.e., $L \ll \lambda_{c}$ ) that $\left(z_{c} / z_{t}\right) \sin \left(k_{c} L\right) \ll 1$ and $\cos \left(k_{c} L\right) \cong 1$, then the pressure amplitude reflection coefficient is the same as (11.9), so $R \cong\left(z_{t}-z_{i}\right) /\left(z_{t}+z_{i}\right)$, making the diaphragm effectively transparent and the pressure reflection coefficient dependent only upon the relative specific acoustic impedances of the gases.

Let us consider a limp latex diaphragm, having a thickness of $0.006^{\prime \prime}=150 \mu \mathrm{m}$, separating air from molecular hydrogen, $\mathrm{H}_{2}$. At $1.0 \mathrm{kHz}$, with $c_{\text {latex }} \cong 1000 \mathrm{~m} / \mathrm{s},\left(k_{c} L\right)=\omega L / c_{\text {latex }} \cong 0.001$. Using $(\rho c)_{\text {latex }}$ $\cong 50 \mathrm{kPa}-\mathrm{s} / \mathrm{m},\left(z_{c} / z_{t}\right) \sin \left(k_{c} L\right) \cong 0.1 \ll 1$, the simplified reflection coefficient of Eq. (11.9) will provide a decent approximation.

For ideal gases, $(\rho c)_{g a s}=\sqrt{\gamma p_{m} \rho_{m}}$. Since the diaphragm is assumed to be limp, the pressures of the gases on either side must be identical (otherwise the limp membrane would bulge and then burst), so $\left(z_{t} / z_{i}\right)_{\text {gas }}=\sqrt{M_{t} / M_{i}}$, where $M$ is the molecular mass of each gas. For air and hydrogen, the ratio of specific acoustic impedances is about $\sqrt{15}$, or its reciprocal, if the planewave originates on the air side, making $R= \pm 0.59$, with the sign dependent upon whether the planewave originates on the air side $(+)$ or the $\mathrm{H}_{2}$ side (-).

\subsubsection{An Impedance Matching Antireflective Layer}

An important special case covered by Eq. (11.12) occurs when the thickness of the intermediate layer is one-quarter of a wavelength to create a perfectly transmitting condition that repeats for integer multiples of half-wavelengths being added to the original quarter wavelength, resulting in an odd-integer multiple of one-quarter of the wavelength of sound, $L=(2 n-1) \lambda_{c} / 4 ; n=1,2,3, \ldots$; hence, $\left(k_{c} L\right)=(n-1 / 2) \pi$. In that case, $\cos \left(k_{c} L\right) \cong 0$ and $\sin \left(k_{c} L\right)=1$. In addition, if $z_{c}=\sqrt{z_{i} z_{t}}$, then $\mathbf{R}=0$ for frequencies near $f=(n-1 / 2) c_{c} / 2 L$, and the intermediate medium is known as a quarterwavelength impedance matching layer.

In high-quality optics, like camera lenses, this effect is used to make antireflective coatings that optimize the transmission of light through the lens. Since this effect is wavelength-dependent, those optical coatings look magenta when they are optimized for transmission of green light $(520 \mathrm{~nm} \lesssim$ $\left.\lambda_{\text {green }} \lesssim 570 \mathrm{~nm}\right)$, since red $\left(620 \mathrm{~nm} \lesssim \lambda_{\text {red }} \lesssim 740 \mathrm{~nm}\right)$ and blue $\left(450 \mathrm{~nm} \lesssim \lambda_{\text {blue }} \lesssim 495 \mathrm{~nm}\right)$ will have non-zero optical reflection coefficients.

Impedance matching layers are very useful for ultrasonic transducers used in medical imaging to optimize the transmission from the transducer material (typically a piezoelectric ceramic) to flesh and to maximize the return signal's excitation of the transducer during detection.

\subsubsection{The "Mass Law" for Sound Transmission Through Walls}

If we consider a solid wall separating two rooms, both containing air, then $z_{c} \gg z_{i}=z_{t}$. In that case, the power transmission coefficient of Eq. (11.14) is further simplified.

$$
T_{\Pi} \cong\left[\frac{2 z_{i}}{z_{c} \sin \left(k_{c} L\right)}\right]^{2} \quad \text { if } \quad z_{c} \gg z_{i}=z_{t}
$$

For reasonably small wall thicknesses, $L \ll \lambda_{c}=c_{c} / f$, $\sin \left(k_{c} L\right) \cong k_{c} L$, so the reduction of sound intensity transmission through the wall depends upon the surface mass density of the wall, $\rho_{S}=\rho_{c} L$. 


$$
T_{\Pi} \cong\left(\frac{2}{k_{c} L} \frac{z_{i}}{z_{c}}\right)^{2} \cong\left[\frac{z_{i}}{\pi f\left(\rho_{c} L\right)}\right]^{2} \quad \text { if } \quad k_{c} L \ll 1
$$

For this reason, in architectural acoustics, this result is known as the "mass law" and is particularly important for multi-occupant dwellings (e.g., apartment buildings) because the transmission of sound depends inversely on the square of both the surface mass density of the wall and of the frequency of the sound. ${ }^{2}$ [2] This inverse-square dependence upon frequency explains why the bass from a neighbor's stereo in an adjoining apartment is more annoying than conversation.

\subsubsection{Duct Constriction/Expansion Low-Pass Filters}

We can exploit Eq. (11.14) for the power transmission coefficient to analyze the one-dimensional propagation through a duct that has an expansion chamber or constriction as shown in Fig. 11.4. Following the analysis in Sect. 10.8, and recognizing that such a system has the same sound speed and fluid density throughout, we can copy the five-wave procedure used in Sect. 11.2, but require the continuity of volume velocity, instead of particle velocity, to produce the analog of Eq. (11.14) where $A$ replaces $z_{i}=z_{t}$ and $A_{c}$ replaces $z_{c}$ [3].

$$
T_{\Pi}=\frac{1}{1+\frac{\left[\left(A_{c} / A\right)-\left(A / A_{c}\right)\right]^{2}}{4} \sin ^{2}(k L)}
$$

It is worthwhile to notice that this coefficient for the power transmission is symmetric with respect to the area ratio, $A_{c} / A$, and its reciprocal, $A / A_{c}$. That means that the result does not depend upon whether the area change is produced by an expansion chamber, $A_{c} / A>1$, shown in Fig. 11.4 (left), or by a constriction, $A / A_{c}>1$, shown in Fig. 11.4 (right).

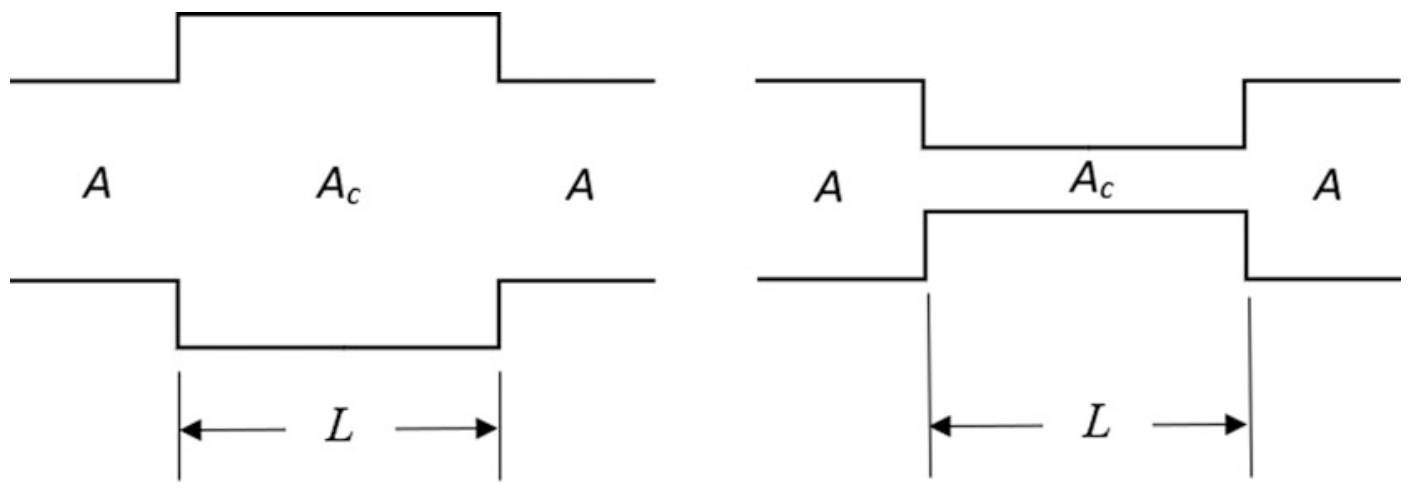

Fig. 11.4 (Left) A compliance with volume, $V=A_{c} L$, is placed in a tube of otherwise uniform cross-sectional area, $A$, to create a low-pass filter. (Right) A constriction with cross-sectional area, $A_{c}<A$, is placed in a tube of otherwise uniform cross-sectional area, $A$, to produce a low-pass filter

\footnotetext{
${ }^{2}$ It is important to remember that this "mass law" is a low-frequency result, as indicated in Eq. (11.16). At higher frequencies, other effects can become significant.
} 


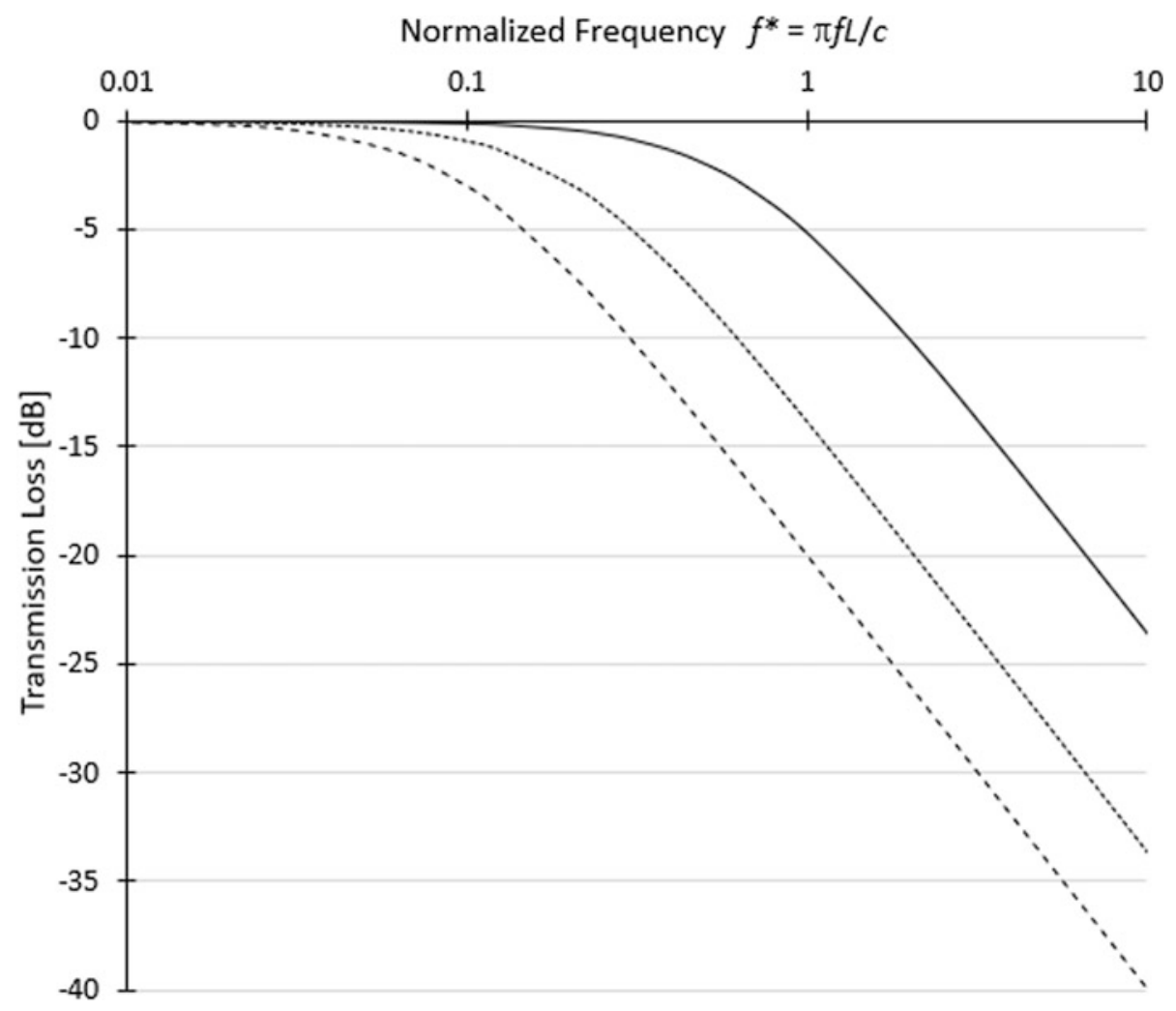

Fig. 11.5 The expansion/constriction duct transmission loss, $\mathrm{T}_{\Pi}$, for small $k L$, in $\mathrm{dB}=10 \log _{10} T_{\Pi}$ for area ratios of $A_{c} / A=2$ or 0.5 (solid line), $A_{\mathrm{c}} / A=5$ or 0.2 (dotted line), and $A_{c} / A=10$ or 0.1 (dashed line) plotted against a normalized frequency, $f^{*}$, that is scaled by the length of the length of the expansion or constriction, $L$, and the sound speed, $c$ : $f^{*}=\pi f L / c$, which is also $f^{*}=\pi L / \lambda=k L / 2$

If we start by examining the limit where $L \ll \lambda$ or $k L \ll 1$, we see that either the expansion chamber or the constriction acts as a low-pass filter as shown in Fig. 11.5 for $A_{c} / A=2, A_{c} / A=5$, and $A_{c} / A=10$.

$$
T_{\Pi}=\left\{1+\frac{\pi^{2} L^{2}}{c^{2}}\left[\left(A_{c} / A\right)-\left(A / A_{c}\right)\right]^{2} f^{2}\right\}^{-1} \quad \text { for } \quad k L \ll 1
$$

As expected from Eq. (11.18), the transmission for $k L<1$ is reduced by $20 \mathrm{~dB} / \mathrm{decade}$, in accordance with the $f^{-2}$ frequency dependence. It is clear from Fig. 11.5 that the frequency at which the transmission is reduced by $3 \mathrm{~dB}$ is dependent upon the area ratio. For $A_{c} / A=2$ or 0.5 , $f^{*}{ }_{-3 \mathrm{~dB}}=0.665$; for $A_{c} / A=5$ or $0.2, f^{*}{ }_{-3 \mathrm{~dB}}=0.208$; and for $A_{c} / A=10$ or $0.1, f^{*}{ }_{-3 \mathrm{~dB}}=0.101$.

As frequency increases, the requirement that $k L<1$ will be violated. In Fig. 11.5, that means that $f^{*} \leq 1 / 2$. This restriction indicates that the frequency bandwidth of the expansion/constriction strategy is rather limited as a low-pass filter. From Eq. (11.17), we see that the transmission coefficient becomes one (i.e., $0 \mathrm{~dB}$, no attenuation), when $k L=\pi$ or $f^{*}=\pi / 2$, and returns to one for all successive $k L=n \pi$ as shown in Fig. 11.6. The maximum transmission loss, $\left(T_{\Pi}\right)_{\max }$, occurs when $L$ is equal to an odd multiple of quarter wavelength, $(2 n-1) \lambda / 4$, making $f=(2 n-1) c / 4 L$.

$$
\left(T_{\Pi}\right)_{\max } \cong-10 \log _{10}\left[(1 / 4)\left(\frac{A_{c}}{A}+\frac{A}{A_{c}}\right)^{2}\right]
$$

For $A_{c} / A=5$ or $0.2,\left(T_{\Pi}\right)_{\max }=-8.3 \mathrm{~dB}$; and for $A_{c} / A=10$ or $0.1,\left(T_{\Pi}\right)_{\max }=-14.1 \mathrm{~dB}$. 


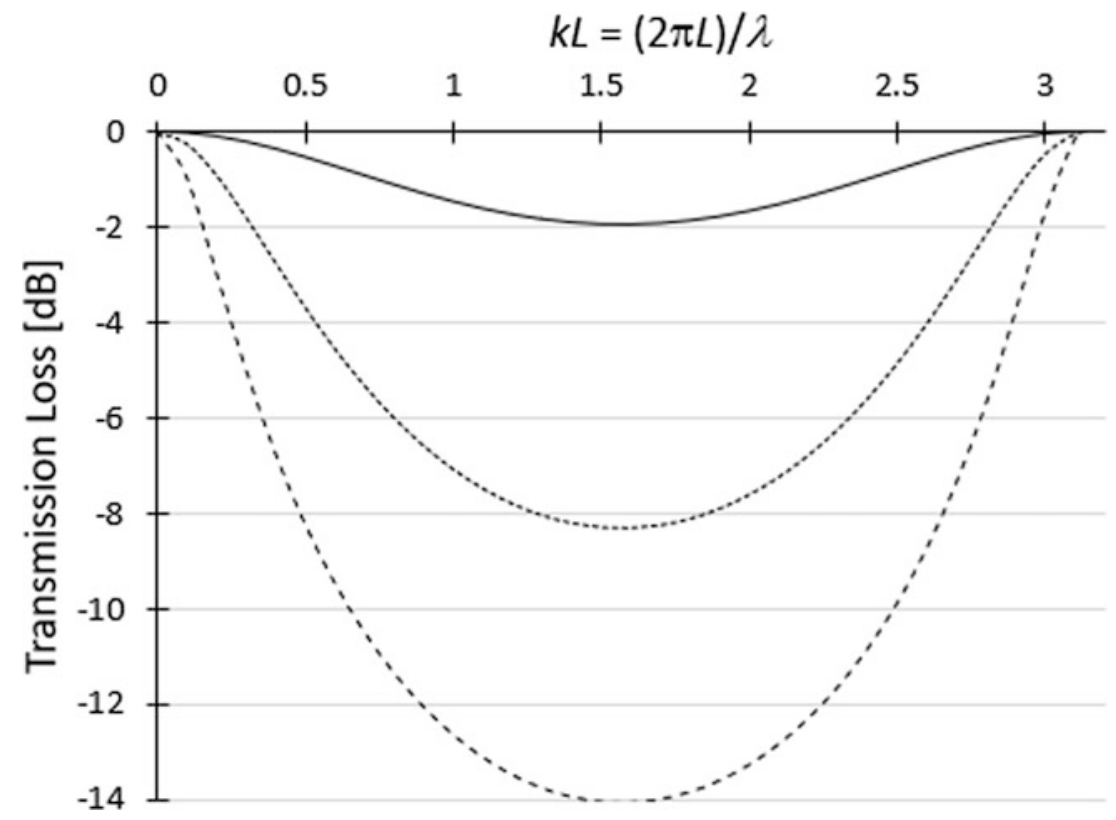

Fig. 11.6 The expansion/constriction duct transmission loss, $\mathrm{T}_{\Pi}$, in $\mathrm{dB}=10 \log _{10} T_{\Pi}$. Shown are area ratios of $A_{c} / A=2$ or 0.5 (solid line), $A_{\mathrm{c}} / A=5$ or 0.2 (dotted line), and $A_{c} / A=10$ or 0.1 (dashed line) plotted against a normalized frequency, $0 \leq k L=(2 \pi L / \lambda) \leq \pi$. This behavior repeats for integer multiples of $\pi$ with no loss for $k L=0$ or $\pi$ and maximum loss for $k L=\pi / 2$

As will be shown in Sect. 13.5.4, for frequencies above $f_{1,1}=0.578 \mathrm{cla}$, where $a=\sqrt{A / \pi}$ is the radius of the circular duct, the planewave nature of propagation in the duct can no longer be guaranteed. That further limits the utility of such a strategy. Despite these limitations, elaborate combinations of expansion chambers and constrictions are used to attenuate sound in automotive exhaust mufflers using techniques that go well beyond those discussed here [4].

\subsection{Snell's Law and Fermat's Principle}

The previous results for planewaves normally incident on an interface between two media can be extended to planewaves that impinge on the interface from some arbitrary angle, $\theta_{i}$, with respect to the normal direction that characterized the surface. The incident wavevector will now have two components, $\vec{k}_{i}=k_{y} \widehat{e}_{y}+k_{x} \widehat{e}_{x}$, where $\widehat{e}_{y}$ and $\widehat{e}_{x}$ are unit vectors in the $+y$ and $+x$ directions.

$$
\begin{aligned}
& p_{i}(x, y, t)=\mathfrak{R} e\left[\widehat{\mathbf{p}}_{\mathbf{i}} \exp j\left(\omega t+\left|\vec{k}_{i}\right| y \cos \theta_{i}-\left|\vec{k}_{i}\right| x \sin \theta_{i}\right)\right] \\
& p_{r}(x, y, t)=\mathfrak{R} e\left[\widehat{\mathbf{p}}_{\mathbf{r}} \exp j\left(\omega t-\left|\vec{k}_{i}\right| y \cos \theta_{r}-\left|\vec{k}_{i}\right| x \sin \theta_{r}\right)\right] \\
& p_{t}(x, y, t)=\mathfrak{R} e\left[\widehat{\mathbf{p}}_{\mathbf{t}} \exp j\left(\omega t+\left|\vec{k}_{t}\right| y \cos \theta_{i}-\left|\vec{k}_{t}\right| x \sin \theta_{t}\right)\right]
\end{aligned}
$$

We can make a simple geometrical argument regarding the directions of the reflected and transmitted planewaves by adopting a perspective that was introduced by the Dutch physicist, Christiaan 


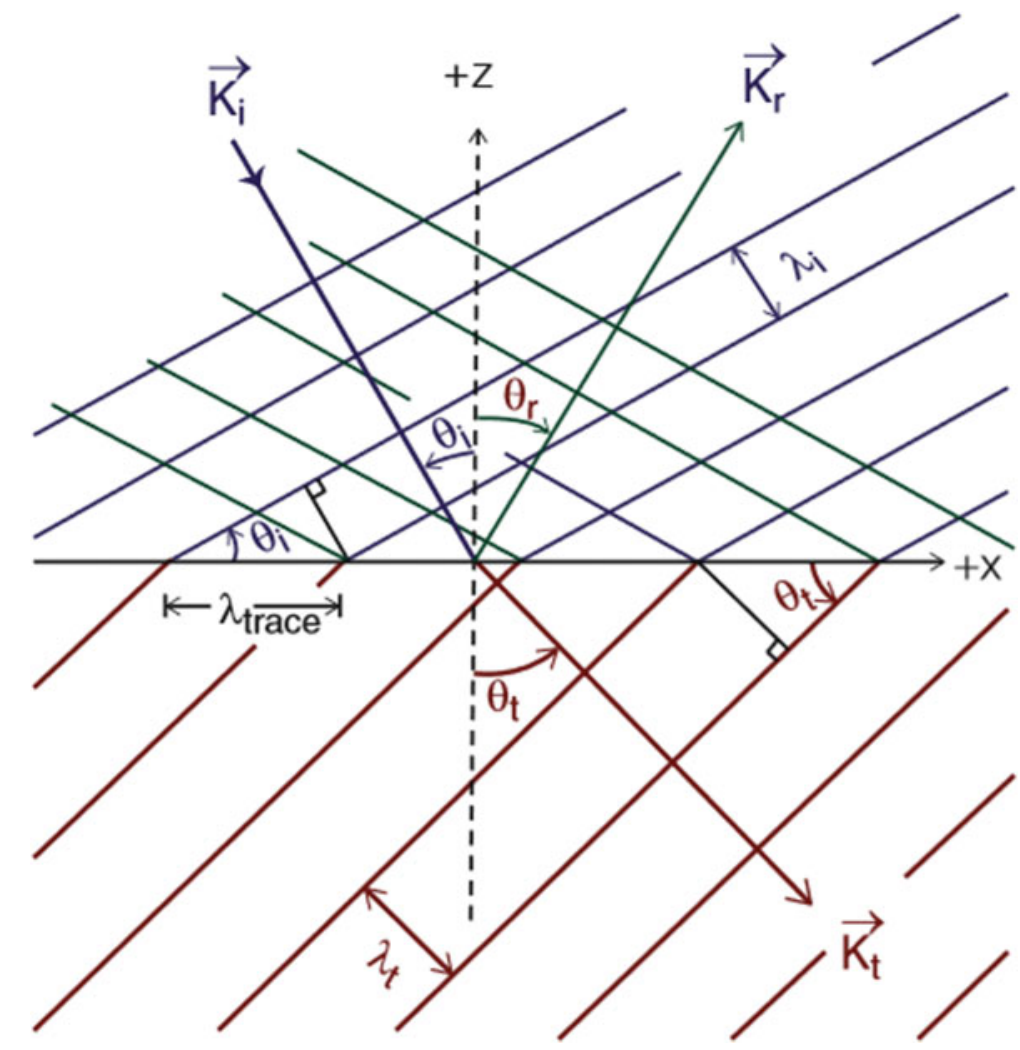

Fig. 11.7 Diagram showing an incident wave with wavevector, $\vec{k}_{i}$, intersecting a plane interface at an angle, $\theta_{i}$, relative to the normal to the interface. Wave fronts of equal phase are shown perpendicular to the wavevector spaced by one wavelength of the incident sound, $\lambda_{i}$. That incident wave excites a reflected wave (green) and a transmitted wave (red). Successive maxima of pressure along the interface are separated by the "trace wavelength," $\lambda_{\text {trace }}$, that is common to all three waves. Based on the wavelengths in this figure, the sound speed in the lower medium, $c_{t}$, is greater than the sound speed in the upper medium, $c_{i}<c_{t}$. When the incident angle equals the critical angle, $\theta_{i}=\theta_{c r i t}$, then the direction of $\vec{k}_{t}$ is along the interface (i.e., $\theta_{t}=90^{\circ}$ ), and we have total internal reflection for all $\theta_{i} \geq \theta_{\text {crit }}$, so no energy is transmitted

Huygens, in $1678,{ }^{3}$ that makes a simple argument which matches boundary conditions at the interface. Figure 11.7 shows the incident wavevector, $\vec{k}_{i}$, with lines normal to $\vec{k}_{i}$, indicating the planes of constant pressure phase. We will imagine those lines represent the pressure maxima of the waves propagating toward the interface. Those wave fronts impinge on the interface, and we will consider each intersection of those incident wave fronts and the interface to be a source of pressure maxima for both the reflected and transmitted planewaves.

The spacing between those maxima along the interface, which we will call the trace wavelength, $\lambda_{\text {trace }}$, is easy to calculate from trigonometry and Garrett's First Law of Geometry ${ }^{4}: \sin \theta_{i}=\lambda_{i} / \lambda_{\text {trace }}$. For normally incident planewaves, the trace velocity $c_{\text {trace }}=f \lambda_{\text {trace }}=\infty$, since the wave fronts of equal phase intersect the interface so that the phase of the pressure on the interface is the same at all locations at every instant. Since $\lambda_{\text {trace }}$ is the same for both the reflected and transmitted waves, the sound speeds

\footnotetext{
${ }^{3}$ C. Huygens, Traite de la Lumiere (completed in 1678, published in Leyden in 1690).

4 "Angles that look alike are alike."
} 
in the two media will determine the angle that the reflected planewave, $\theta_{r}$, and the angle transmitted planewave, $\theta_{t}$, make with the direction of the normal to the interface. ${ }^{5}$

$$
\lambda_{\text {trace }}=\frac{\lambda_{i}}{\sin \theta_{i}}=\frac{\lambda_{r}}{\sin \theta_{r}}=\frac{\lambda_{t}}{\sin \theta_{t}}
$$

The sound speed, $c_{i}$, is the same for both the incident and reflected planewaves, so $\theta_{r}=\theta_{i}$; the angle of incidence equals the angle of reflection. This case is called specular reflection. If the interface has a surface roughness that is characterized by random assortments of "bumps" that are all much smaller in height and extent than the wavelength of sound, the reflection will be diffuse.

Again, in a linear system, the frequency, $\omega$, with which the incident planewave drives the interface, must also be the frequency of the driven reflected and transmitted planewaves. Since $\lambda=c / f$, we can invert Eq. (11.21) to produce a result known as Snell's law. ${ }^{6}$

$$
\frac{\sin \theta_{i}}{c_{i}}=\frac{\sin \theta_{r}}{c_{i}}=\frac{\sin \theta_{t}}{c_{t}}
$$

Before moving on to calculation of the pressure reflection and transmission coefficients, it worth noticing that Snell's law could have been derived by insisting that propagation time from one location in the upper medium to some other location in the lower medium is minimized by the same condition on the angles that is expressed in Eq. (11.22). This approach utilizes Fermat's principle (1622) $)^{7}$ : "The actual path between two points taken by a [wave] is the one that is traversed in the least time."

For the actual path to be an extremum of the total transit time, a neighboring path must take the same time; their time difference being at most second order in the deviation of the alternate path from the path of least time. Figure 11.8 provides a diagram of the path of least time (ACB) and a nearby path (AXB).

In the upper medium of Fig. 11.5, the perpendicular XE makes $\mathrm{CE}$ the reduction in distance traveled by the nearby path AX. The time savings by traveling via $\mathrm{AC}$ is therefore $t_{i}=\mathrm{CE} / c_{i}$. Similarly, in the lower medium, the perpendicular CF makes XF the additional distance traveled by the nearby path $\mathrm{XB}$. The excess time in the lower medium is therefore $t_{t}=\mathrm{XF} / c_{t}$. Since $\measuredangle \mathrm{EXC}=\theta_{i}$ and $\measuredangle \mathrm{FCX}=\theta_{t}$, we can express the two transit times for the nearby path in terms of the common length $\mathrm{XC}$ using simple trigonometry.

$$
t_{i}=\frac{E C}{c_{i}}=\frac{X C \sin \theta_{i}}{c_{i}}=t_{t}=\frac{X F}{c_{t}}=\frac{X C \sin \theta_{t}}{c_{t}}
$$

Equation (11.23) also yields Snell's law after dividing through by XC.

\footnotetext{
${ }^{5}$ Since the trace wavelength is constant, its reciprocal, the trace wavenumber, $2 \pi / \lambda_{\text {trace }}$, is also constant. In the underwater acoustic propagation community, this is commonly called "conservation of the horizontal wavenumber" [12]. Since I associate the term "conservation" with quantities that obey equations like (10.35), I prefer to consider the horizontal wavenumber to be an invariant, like the trace wavelength.

${ }^{6}$ Snell's law is known as Descartes' law in France. Willebrord Snellius (1580-1626) was not the first to produce that result. The law was first accurately described by the Persian scientist, Ibn Sahl, in Baghdad, where in 984, he used the law to derive lens shapes that focus light with no geometric aberrations as described in the manuscript On Burning Mirrors and Lenses.

${ }^{7}$ The principle of least time was actually first applied to reflections from a mirror much earlier, by Hero of Alexandria, around $60 \mathrm{CE}$. Since the reflected light propagates at the same speed as the incident light, this is equivalent to the path that is also the shortest geometrical distance.
} 


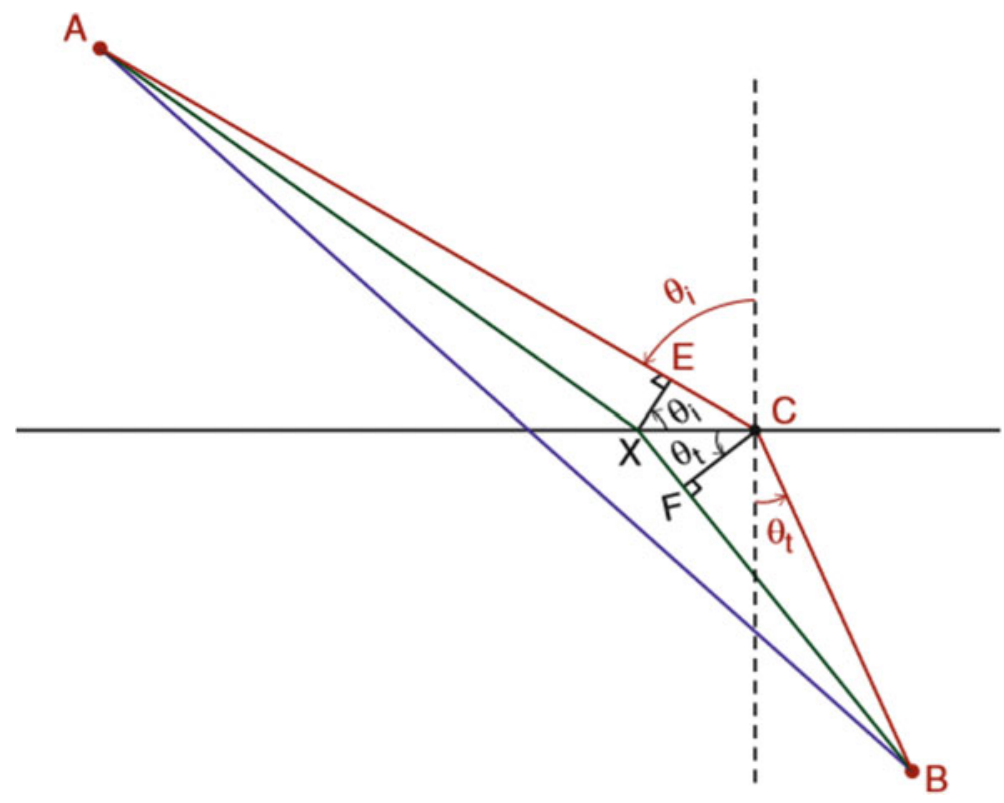

Fig. 11.8 Three paths from point $A$ in the upper medium to point B in the lower medium. In this diagram, it is assumed that the propagation speed of the wave in the lower medium, $c_{t}$, is slower than the propagation speed in the upper medium, $c_{i}>c_{t}$. The path AB (blue) is the shortest distance, and the path ACB (red) takes the least time. The angles $\theta_{i}$ and $\theta_{t}$ can be determined by calculating the additional travel time beyond that required for ACB when going along the path through AXB (green) and setting that excess equal to zero [5]

\subsubsection{Total Internal Reflection}

In passing from a faster medium into a slower medium, $c_{i}>c_{t}$, the angle that the transmitted wavevector makes with respect to the normal at the interface, $\theta_{t}$, is smaller than the incident angle, $\theta_{i}$, also measured from the normal. If the incident medium has a slower sound speed than the transmission medium, $c_{i}<c_{t}$, then it is possible that the angle of transmission, $\theta_{t}$, dictated by Snell's law, could become a complex number. In that case, we can define a critical angle, $\theta_{\text {crit }}$, where $\theta_{t}=90^{\circ}$ (i.e., the transmitted wave travels along the interface but not into the second medium).

$$
\sin \theta_{c r i t}=\frac{c_{i}}{c_{t}} ; \quad \text { if } \quad c_{i}<c_{t}
$$

This is a consequence of the fact that the minimum trace velocity, $c_{\text {trace }}=f \lambda_{\text {trace }}$, shown in Fig. 11.7, in the lower medium, is limited to $c_{t}$, which equals $c_{\text {trace }}$ when $\theta_{t}=90^{\circ}$. Using the fact that $\cos ^{2} \theta+\sin ^{2} \theta=1$, the angle of transmission can be expressed in terms of the angle of incidence and the sound speed ratio.

$$
\cos \theta_{t}=\sqrt{1-\sin ^{2} \theta_{t}}=\sqrt{1-\left(c_{t} / c_{i}\right)^{2} \sin ^{2} \theta_{i}}=-j \sqrt{\left(c_{t} / c_{i}\right)^{2} \sin ^{2} \theta_{i}-1}
$$

If $c_{t}>c_{i}$, the first term under the square root becomes imaginary, which is expressed explicitly in the final term at the right of Eq. (11.25).

Substitution of Eq. (11.25) into the expression for the transmitted pressure in Eq. (11.20) produces an exponential decay of the sound with increasing distance away from the interface, for incident angles that exceed the critical angle, producing an inverse characteristic depth, $(1 / \delta)$. 


$$
\begin{gathered}
k_{z}=\frac{1}{\delta}=\left|\vec{k}_{t}\right| \sqrt{\left(c_{t} / c_{i}\right)^{2} \sin ^{2} \theta_{i}-1} \\
p_{t}(x, z, t)=\mathfrak{R} e\left[\widehat{\mathbf{p}}_{\mathbf{t}} e^{+(y / \delta)} \mathrm{e}^{j\left[\omega t-\left|\vec{k}_{i}\right| x \sin \theta_{i}\right]}\right] ; \quad \text { for } y \leq 0
\end{gathered}
$$

This solution is a wave that is localized just below the interface. For angles of incidence, $\theta_{i}<\theta_{\text {crit }}$, the transmitted wave fills the half-space of the lower medium, as shown in the lower half of Fig. 11.7. When $\theta_{i}=\theta_{\text {crit }}$, all of that outgoing energy collapses to the surface in a layer with exponential thickness, $\delta$. The thickness of that layer decreases monotonically as $\theta_{i}$ increases beyond $\theta_{\text {crit }}$.

If we apply the Euler equation to Eq. (11.27) to determine the velocity, $v_{t}$, normal to the surface, we find $\widehat{\mathbf{v}}_{\mathbf{t}}=j \widehat{\mathbf{p}}_{\mathbf{t}}(\omega \rho \delta)$, so the pressure and particle velocity are $90^{\circ}$ out-of-phase. The intensity leaving the interface is zero since there is no in-phase component of pressure and velocity. That does not mean that the layer contains no energy, just that none of the energy is propagating. If there were some scattering centers (i.e., defects, voids, inclusions of material with some compressibility, and/or density contrast), the energy trapped in that layer could be reradiated (i.e., scattered; see Sect. 12.6) and escape.

The concept of total internal reflection was first recognized in optics. One application of total internal reflection that I find particularly inspiring occurs in the compound eyes of insects and other arthropods. With thousands of lenses, the use of an equal number of irises, like those in avian and mammalian eyes, is not particularly efficient. In the arthropod's compound eye, each lens is connected to the optic nerve by a transparent tube that contains the light by total internal reflection, due to the difference between the index of refraction (i.e., the optical equivalent of our specific acoustic impedance) between the tube and the surrounding fluid. The way that insects control the amount of light that reaches the optic nerve is by a chemical reaction in the fluid that surrounds the tube. Under bright lighting, the fluid will produce a precipitate with small particles that scatters light out of the trapped layer, thereby reducing the amount of light reaching the optic nerve. The physical structure of the compound insect eye is shown in Fig. 11.9.

Proof of this system's efficacy is the fly's ability to avoid getting swatted outdoors in broad daylight and indoors in a darkened room. (We should now pause briefly out of respect for Darwin and the power
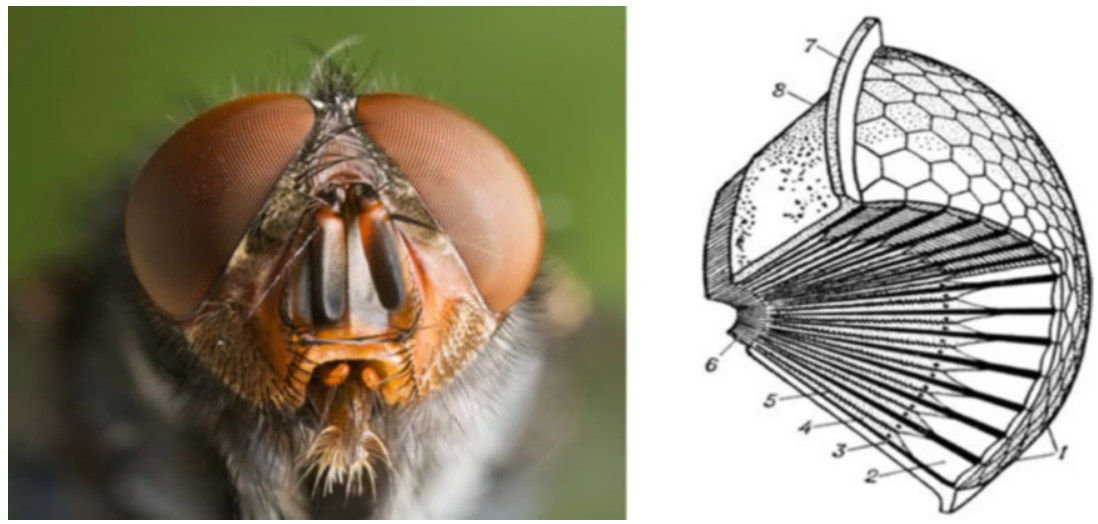

Fig. 11.9 (Left) The compound eyes of the blue bottle fly. (Right) Each of the "tubes" is optical fibers, called an ommatidium. They guide light from the lens to the optic nerve endings (4 and 5) using total internal reflection to regulate the transmission of the light. Ant eyes have about six ommatidia and dragonfly eyes have as many as 25,000 . This anatomical drawing of a compound eye shows the corneal lens (1) and the crystalline cone (2) which combine to form the dioptric apparatus. At the base is the light-sensitive rhabdom (5 and 6). The space between the tubes (3) contains the pigment cells that will form a precipitate that scatters some of the optical energy out of the trapped layer at the interface if the light is too bright, effectively reducing the field of view (known in optics as the "numerical aperture" of the lens). (Photo and diagram taken from Wikipedia) 
of natural selection.) I would be most interested in finding a similar system for controlling the sound amplitude that reaches the auditory nerve in any animal, although the ability to focus sound seems ubiquitous in marine mammals, as shown for the Cuvier's beaked whale in Fig. 11.18.

\subsubsection{The Rayleigh Reflection Coefficient}

Although we have calculated the angles of reflection and transmission for planewaves of sound impinging on a planar interface from an arbitrary direction, we have yet to calculate the amplitude reflection coefficient in terms of the specific acoustic impedances, as we did for the case of normal incidence at the interface between two media in Eq. (11.9). To produce the equivalent of Eq. (11.9) for oblique incidence (i.e., $\theta_{i} \neq 0^{\circ}$ ), we need to generalize the velocity boundary condition of Eq. (11.5), since it is only the normal component of the velocity that needs to be continuous across the boundary to avoid cavitation at the interface. ${ }^{8}$

$$
\left|\widehat{\mathbf{v}}_{\mathbf{i}}\right| \cos \theta_{i}+\left|\widehat{\mathbf{v}}_{\mathbf{r}}\right| \cos \theta_{r}=\left|\widehat{\mathbf{v}}_{\mathbf{t}}\right| \cos \theta_{t}
$$

Pressure continuity, expressed in Eq. (11.6), is unchanged since pressure is a scalar quantity.

Forming the ratio of those boundary conditions, as we did in Eq. (11.7), leads to the desired result for the ratio of the reflected pressure to the incident pressure $R \equiv \widehat{\mathbf{p}}_{\mathbf{r}} / \widehat{\mathbf{p}}_{\mathbf{i}}$.

$$
R \equiv \frac{\widehat{\mathbf{p}}_{\mathbf{r}}}{\widehat{\mathbf{p}}_{\mathbf{i}}}=\frac{\frac{z}{\cos \theta_{t}}-\frac{z_{i}}{\cos \theta_{i}}}{\frac{z_{t}}{\cos \theta_{t}}+\frac{z_{i}}{\cos \theta_{i}}}
$$

Once again, we are confronted with an expression for the reflection coefficient that can vanish, providing perfect transmission, if the numerator of Eq. (11.29) is zero.

$$
\frac{\cos \theta_{t}}{\cos \theta_{i}}=\frac{z_{t}}{z_{i}}
$$

Using Snell's law to eliminate $\theta_{t}$, this angle, called the angle of intromission, $\theta_{\text {intro }}$, can be expressed in terms of both the impedance and sound speed or mass density ratios.

$$
\sin ^{2}\left(\theta_{\text {intro }}\right)=\frac{\left(z_{t} / z_{i}\right)^{2}-1}{\left(z_{t} / z_{i}\right)^{2}-\left(c_{t} / c_{i}\right)^{2}}=\frac{1-\left(z_{i} / z_{t}\right)^{2}}{1-\left(\rho_{1} / \rho_{2}\right)^{2}}
$$

Not every combination of media will have an angle of intromission that depends upon both sound speed and density ratios. For $\theta_{\text {intro }}$ to be real, both the numerator and denominator of Eq. (11.31) must have the same sign.

There are four combinations of impedance and sound speed ratios:

(i) No $\boldsymbol{\theta}_{\text {crit }}$ and $\boldsymbol{n o} \boldsymbol{\theta}_{\text {intro }}$ If both $z_{t} / z_{i}<1$ and $c_{t} / c_{i}<1$, there is no critical angle, so $0<R<-1$ for all $\theta_{i}$. For this case, $\theta_{i}>\theta_{t}$, so $\left(\cos \theta_{t} / \cos \theta_{i}\right)>1$ and $z_{t} / z_{i}<1$, so there can be no angle of intromission.

\footnotetext{
${ }^{8}$ If we were treating the interface between two viscous liquids or between a viscous liquid and a solid surface, then we would need to impose a "no-slip" boundary condition on the transverse components of particle velocity. This is particularly important for the fluid-solid boundary since the viscous stress on the interface can couple to shear waves into the solid and vice versa. The coupling of shear waves in the solid to viscous waves in liquids has been used to measure fluid density and viscosity.
} 
(ii) Real $\boldsymbol{\theta}_{\text {crit }}$ but no $\boldsymbol{\theta}_{\text {intro }}$ If both $z_{t} / z_{i}>1$ and $c_{t} / c_{i}>z_{t} / z_{i}$, then there will be a critical angle above which there will be total internal reflection so $R=1$ if $\theta_{i}>\theta_{\text {crit }}$. Since $c_{t} / c_{i}>z_{t} / z_{i}$, the numerator of Eq. (11.31) will be positive, but the denominator will be negative, so there can be no $\theta_{\text {intro }}$.

(iii) Real $\boldsymbol{\theta}_{\text {intro }}$ but no $\boldsymbol{\theta}_{\text {crit }}$ If $z_{t} / z_{i}>1$ and $c_{t} / c_{i}<1$, then there will be no critical angle, so $0 \leq R<1$ for all $\theta_{i}$. Since $c_{t} / c_{i}<z_{t} / z_{i}$, both the numerator and the denominator of Eq. (11.31) will be positive, so there will be an angle of intromission, $\theta_{i}=\theta_{\text {intro }}<90^{\circ}$.

(iv) Real $\boldsymbol{\theta}_{\text {intro }}$ and real $\boldsymbol{\theta}_{\text {crit }}$ If $c_{t} / c_{i}>1$ and $z_{t} / z_{i}<1$, then there will be both a critical angle, $\theta_{\text {crit }}<90^{\circ}$, above which there will be total internal reflection so $R=1$ if $\theta_{i}>\theta_{\text {crit }}$, and both the numerator and the denominator of Eq. (11.31) will be negative, so there will be an angle of intromission $\theta_{i}=\theta_{\text {intro }}<\theta_{\text {crit }}<90^{\circ}$.

Total transmission at the angle of intromission has been observed in high-porosity marine sediments, like silty clays, which exhibit a sound speed through its bulk which is lower than that of the interstitial fluid within its pores. When high-porosity sediment is at the water/sediment interface, there can be total transmission of sound into the seafloor. Measurements of the angle of intromission in coastal regions around Italy indicate that the properties of the high-porosity sediments are surprisingly uniform over large areas [6].

\subsection{Constant Sound Speed Gradients}

To this point in our discussion of the refraction of sound, we have only considered plane boundaries that separate media with a discontinuity in their sound speed, density, and/or specific acoustic impedance. There are many situations of interest where the refraction (or bending) of a planewave of sound can create interesting and significant effects resulting from a gradual change in the acoustical properties of the medium. These effects are easily calculable in the limit that such sound speed changes are a linear function of position when those changes occur over a distance that extends over very many acoustic wavelengths.

Figure 11.10 shows two examples of sound refraction in air that supports a temperature gradient that depends upon the time of day. As expressed by the quadratic dependence of sound speed on the mean temperature of an ideal gas in Eq. (10.23), the change in sound speed, $\Delta c$, with height, $z$, above the ground is related to the changes in the absolute temperature, $T$.

$$
\frac{d c}{d z}=\left(\frac{d c}{d T}\right)\left(\frac{d T}{d z}\right)=\frac{c}{2 T}\left(\frac{d T}{d z}\right)
$$

Similar refractive effects occur in the ocean where the speed of sound in seawater is a complicated function of temperature, salinity, and depth (pressure).

$$
\begin{aligned}
c= & 1493.0+3(t-10)-0.006(t-10)^{2}-0.04(t-18)^{2} \\
& +1.2(S-35)-0.01(t-18)(S-35)+D / 61
\end{aligned}
$$

In Eq. (11.33), the sound speed, $c$ in $\mathrm{m} / \mathrm{s}$, is a function of the temperature, $t$, in degrees Celsius. The salinity, $S$, is measured in grams of salt per kilogram of water, and $D$ is the depth below the surface measured in meters [8]. That formula is valid within $\pm 0.2 \mathrm{~m} / \mathrm{s}$ for $-2{ }^{\circ} \mathrm{C} \leq t \leq+24.5{ }^{\circ} \mathrm{C}$, $0.030 \leq S \leq 0.042$, and $0 \leq D \leq 1000 \mathrm{~m}$.

Figure 11.11 shows the sound speed variation with depth that was measured over 9 years in the ocean 15 miles $(24 \mathrm{~km})$ south-east of Bermuda. Although significant sound speed variation over the 9 years is apparent within roughly $2 \mathrm{~km}$ of the surface, it is possible to represent the change in sound speed with depth using a piecewise-linear approximation. 

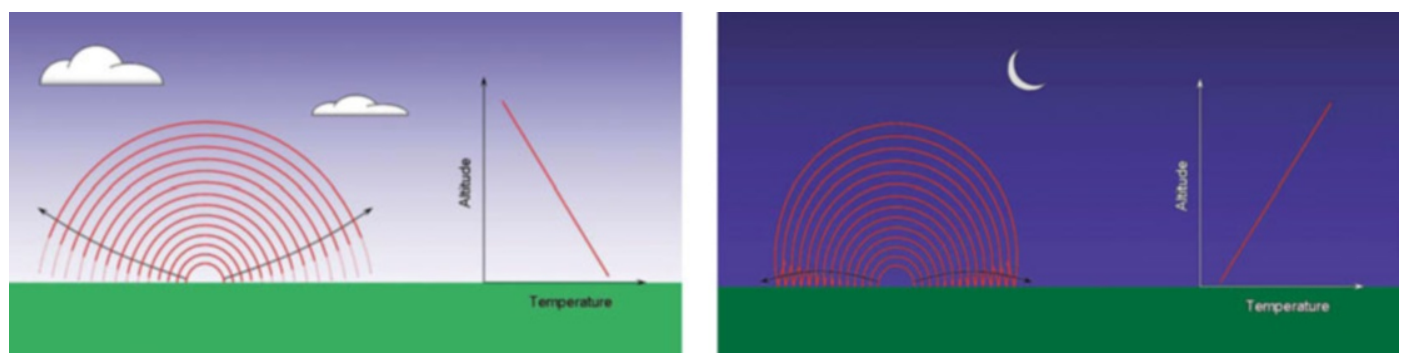

Fig. 11.10 (Left) During the day, the temperature of the atmosphere generally decreases with altitude above the ground causing sound waves to refract upward since the speed of sound is proportional to $\sqrt{T}$. (Right) The opposite conditions can occur at night when the temperature of the air in contact with the ground is colder that than the air above it (a "temperature inversion"). This causes sound to be refracted downward. If there is wind, it can also cause the sound speed to vary with height above the ground. (Figures courtesy of T. B. Gabrielson [7])
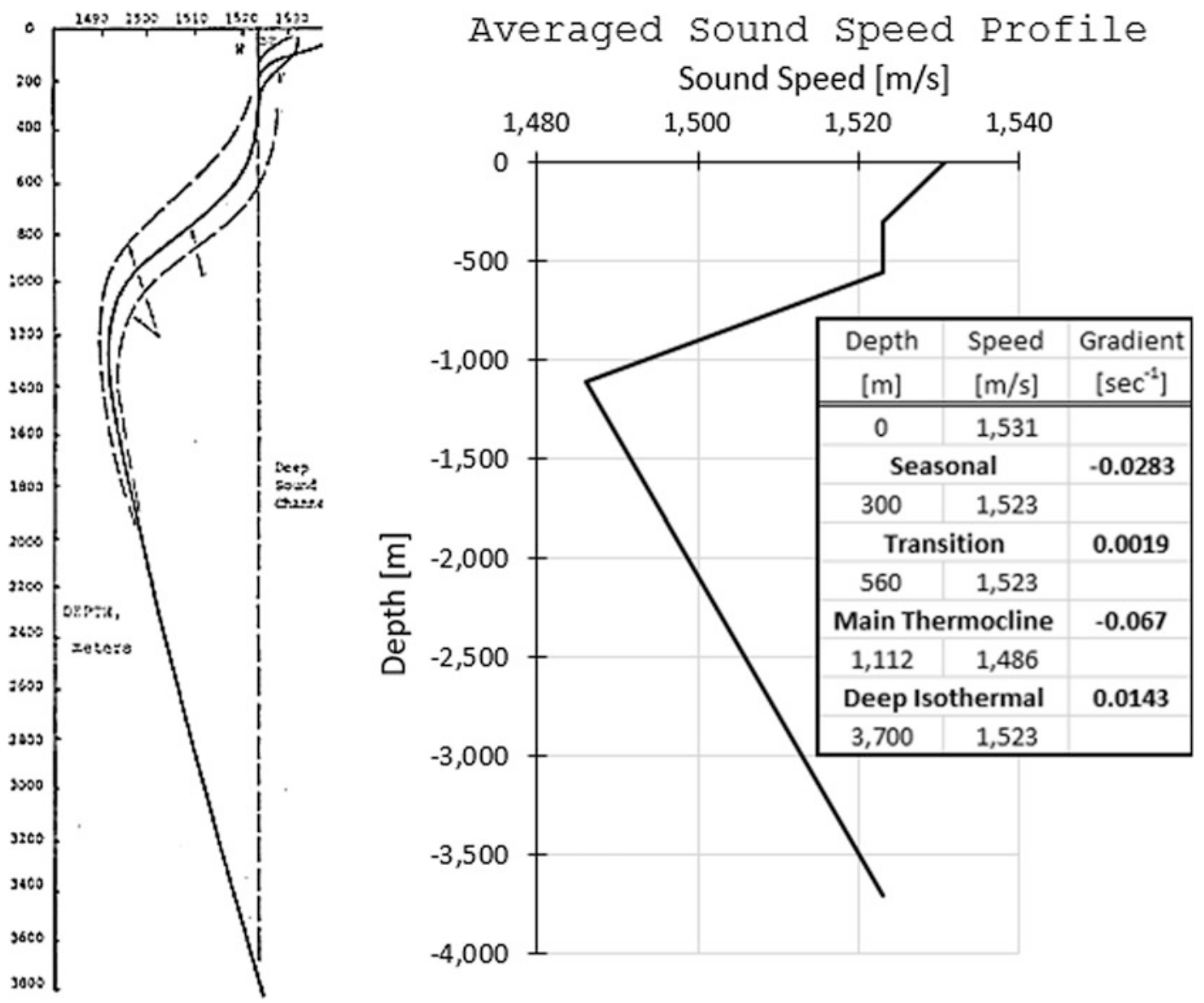

Fig. 11.11 (Left) These deep-ocean sound speed profiles were taken every 2 weeks over a 9-year period at a location $24 \mathrm{~km} \mathrm{SE}$ of Bermuda. [9] The solid curve is the average and the dashed curves show the extremes. This typical deep-sea profile may be divided into a number of layers having different properties. At the top, the diurnal layer shows day-night variability and responds to weather changes. Below it, down to the depth of about $300 \mathrm{~m}$, lays the seasonal thermocline that is above the main thermocline, which exhibits the strongest sound speed gradient. Below the thermocline, beneath $1200 \mathrm{~m}$, the deep isothermal layer has a constant temperature of $+4{ }^{\circ} \mathrm{C}$. There, the sound speed's increase with depth is dominated by the increasing pressure. (Right) The measured sound speed profile is replaced by a piecewise-linear approximation that provides a constant sound speed gradient for each layer, as indicated by the embedded table. At a depth of approximately $3700 \mathrm{~m}$, the sound speed is the same as it is at a depth of approximately $560 \mathrm{~m}$. Between those two depths, sound can be trapped in the "deep sound channel" in the same way that light is trapped in an optical fiber, except the core of an optical fiber that is about $10 \mu \mathrm{m}$ and the sound channel that is about $1 \mathrm{~km}$, a height ratio of about $10^{8}$ 


\subsubsection{Constant Gradient's Equivalence to Solid Body Rotation}

The goal in this sub-section is to develop a simple formalism that will permit calculation of the path of planewaves in a medium with a sound speed that is a linear function of height or depth. Furthermore, we would like to be able to track the trajectory of the sound (i.e., follow the direction of the wavevector) through regions of changing sound speed gradient, a process known as ray tracing. To do this, we must first recognize that the propagation of sound through a region of constant sound speed gradient is isomorphic to the solid body rotation of a disk, since the tangential velocity, $\vec{v}_{\text {tan }}$, of any point on the rotating disk is proportional to its (linear) distance, $|\vec{r}|$, from the axis of rotation.

$$
\vec{v}_{\tan }=\vec{r} \times \vec{\omega}
$$

Since this equation refers to a rotating disk, the use of the cross-product might seem to be pedantic excess, but it is intended to remind you of your study of solid body rotational dynamics during your freshman physics course.

If we limit ourselves to linear changes in sound speed with height or depth, then the sound speed gradient is a constant: $\mathrm{d} c / \mathrm{d} z \equiv g$. In analogy with a rotating disk of radius, $R$, as diagrammed in Fig. 11.12, we can equate sound speed, $c$, with the tangential velocity of a point on the rim of the disk, $\vec{v}_{\text {rim }}$, and integrate our above definition; it is easy to see that $g$ plays the same role as $\omega$ (and has the same units) in Eq. (11.34).

$$
\int_{0}^{v_{r i m}} d c=g \int_{0}^{R} d z \Rightarrow R=\frac{\left|\vec{v}_{\text {rim }}\right|}{g}
$$

I like to think of the refraction (bending) of the sound wave's trajectory in a constant sound speed gradient by picturing the advance of the planewave fronts as represented schematically in Fig. 11.13.

Fig. 11.12 Shown at the right is a disk that is rotating. The dashed, colored arrows indicate the tangential velocity that increases linearly with distance from the axis of rotation. Such a velocity distribution in space is identical to the assumed constant sound speed gradient that will control the refraction of sound waves

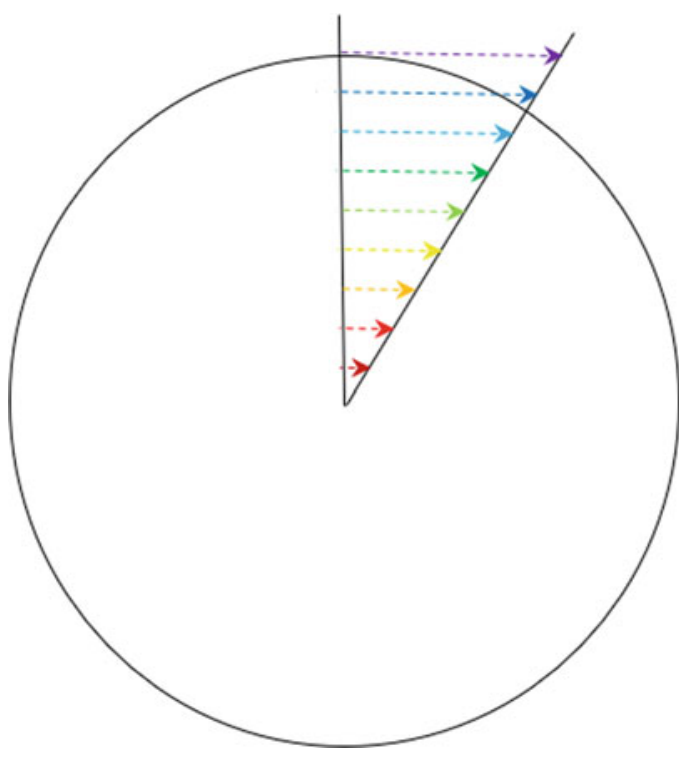




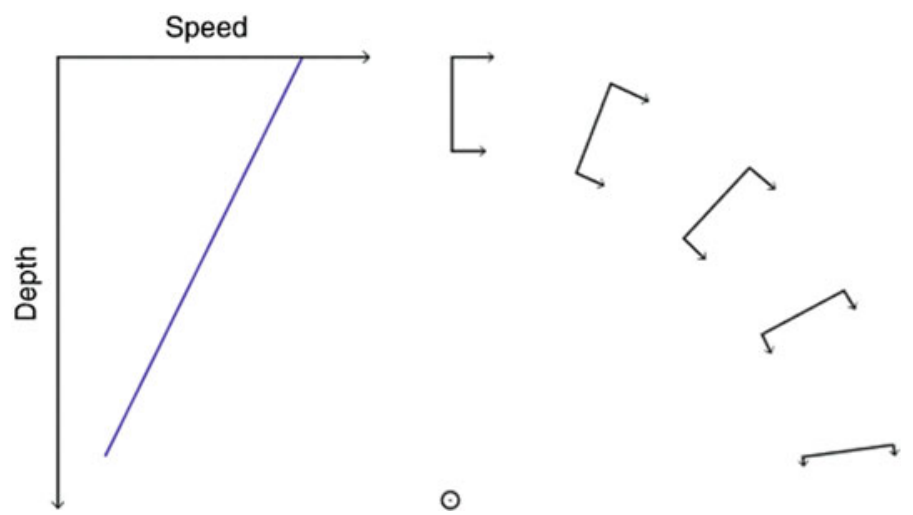

Fig. 11.13 A conceptual sketch of planewave fronts moving through a medium with the velocity gradient shown at the left. The arrows at the top of the wave fronts are always longer than the arrows at the bottom. This turns the wave front downward until the wave front is nearly horizontal. Once horizontal, both ends of the wave front will move with the same speed, and the wave will continue downward without changing direction

To introduce this "disk rotation" approach to analysis of the propagation of planewaves through a medium with constant speed gradients, let's consider the measurement of road noise during the day, when the temperature of the air is decreasing with increasing altitude, as shown in Fig. 11.10 (left).

Assume that you are asked to determine the sound pressure level created by traffic on a highway at a proposed housing location that is $150 \mathrm{~m}$ from the highway. By measuring the average temperature at a height of $0.5 \mathrm{~m}$ and at $5 \mathrm{~m}$, you determine that the sound speed at $0.5 \mathrm{~m}$ is $342.8 \mathrm{~m} / \mathrm{s}$ and at $5.0 \mathrm{~m}$ is $341.3 \mathrm{~m} / \mathrm{s}$. How high must your microphone be above the ground, at a distance of $150 \mathrm{~m}$ from the source, to ensure that you intercept sound that leaves the highway in an initially horizontal direction?

To begin, let's calculate the sound speed gradient, $\mathrm{d} c / \mathrm{d} z=g \cong \Delta c / \Delta z=(2.8-1.3 \mathrm{~m} / \mathrm{s}) /$ $4.5 \mathrm{~m}=0.333 \mathrm{~s}^{-1}$. Since the sound speed decreases with height, we know that the wave will be refracted upward, as shown in Fig. 11.10 (left). We'll assume that the noise is generated at the intersection of the tire and the road at $z=0$, where $c_{o}=343.0 \mathrm{~m} / \mathrm{s}$ (extrapolating down to the surface from the lowest measurement at $0.5 \mathrm{~m}$ above the ground).

We now need to calculate the radius, $|R|$, of this limiting ray's circular path.

$$
|R|=\frac{c_{o}}{g}=1029 \mathrm{~m}
$$

A simple trigonometry identity can be used to determine the height, $h=11.0 \mathrm{~m}$, above the ground that a microphone must be placed to receive the sound radiated by the tires. For me, drawing a sketch, like in Fig. 11.14, is always crucial.

$$
\begin{gathered}
\sin \theta=\frac{150}{1029}=0.146 \Rightarrow \theta=0.146 \mathrm{rad}=8.38^{\circ} \\
h=|R|-|R| \cos \theta=|R|(1-\cos \theta) \cong|R| \frac{\theta^{2}}{2}=11.0 \mathrm{~m}
\end{gathered}
$$

The final expression above made use of the small-angle expansion of cosine in Eq. (1.6).

Although the refractive process was not understood until the twentieth century [7], reports of the existence of an acoustic "shadow zone" appeared much earlier. Below is the account of R. G. H. Kean as he watched the Battle of Gaines's Mill during the American Civil War [10]: 


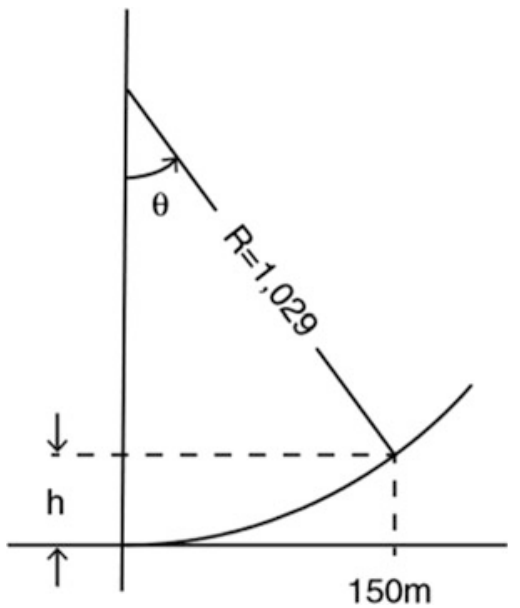

Fig. 11.14 Sketch (not to scale) of the ray path of the sound generated by the tire noise on a road surface. The observation point is $150 \mathrm{~m}$ from the sound source. Between the ground and the circular arc is a "shadow zone" that is created by the upward refraction of the sound produced by the sound speed gradient, $\mathrm{d} c / \mathrm{d} z=0.333 \mathrm{~s}^{-1}$, which was used to calculate the radius, $I R I=1029 \mathrm{~m}$, applying Eq. (11.36). A microphone must be placed at least a distance, $h$, above the ground to intercept the tire noise. At the surface $(h=0)$, the sound speed is $c_{o}=343.0 \mathrm{~m} / \mathrm{s}$. At $h=11.0 \mathrm{~m}$ above the surface, the sound speed is $339.3 \mathrm{~m} / \mathrm{s}$

I distinctly saw the musket-fire of both lines ... I saw batteries of artillery on both sides come into action and fire rapidly. Yet looking for near two hours, from about 5 to 7 P.M. on a midsummer afternoon, at a battle in which at least 50,000 men were actually engaged, and doubtless at least 100 pieces of field-artillery ... not a single sound of the battle was audible to General Randolph and myself ... [However, the] cannonade of that very battle was distinctly heard at Amhurst Court-house, 100 miles west of Richmond, as I have been most credibly informed.

The process of refraction in a sound speed gradient, as illustrated in a particularly simple form in Fig. 11.14, is entirely reversible. If we were to say that we had a sound source directed $8.4^{\circ}$ below the horizontal direction, located $11.0 \mathrm{~m}$ above the ground, then we could just as easily say that it would intersect the ground $150 \mathrm{~m}$ away, based on Fig. 11.14 and the results of Eq. (11.37).

Since much of the interest in the propagation of sound in a sound speed gradient (linear or otherwise) tends to concern sound waves that are nearly grazing the horizontal axis, the grazing angle is more commonly used in Snell's law than the angle measured with respect to the normal to the surface, which was our previous choice for discussion of reflection and transmission from a plane surface of discontinuity. If we now use the grazing angle, then we can rewrite Eq. (11.22) by letting $\theta$ be the angle below the horizontal and $c_{o}$ be the sound speed at a location where the sound waves are propagating horizontally (i.e., $\cos 0^{\circ}=1$ ).

$$
\frac{c}{\cos \theta}=c_{o}
$$

Let's apply the above version of Snell's law to the example in Fig. 11.14. That ray is horizontal at $h=0$, where $c_{o}=343.0 \mathrm{~m} / \mathrm{s}$. Assuming, as before, a constant sound speed gradient, at the "source height" of $11.0 \mathrm{~m}, c=339.3 \mathrm{~m} / \mathrm{s}$. Plugging directly into Eq. (11.38), $\cos \theta=c / c_{o}$, so $\theta=0.147$ radians $=8.4^{\circ}$. Of course, we could have used Eq. (11.37) to calculate the sound speed $11.0 \mathrm{~m}$ above the surface, since we already knew that $\theta=8.4^{\circ}$. 


\subsubsection{Sound Channels}

We will now apply this formalism to a particularly interesting case that occurs in a sound speed profile like the one shown in Fig. 11.11, describing the deep ocean off of Bermuda, which we have approximated by a series of contiguous line segments with constant (but different) sound speed gradients. There is a sound speed minimum of $c_{\min }=1486 \mathrm{~m} / \mathrm{s}$, at a depth of $1112 \mathrm{~m}$, between the main thermocline and the deep isothermal layer. As evident from Fig. 11.13, in such a constant gradient (i.e., linear sound speed profile), the sound will "go to the slow," and therefore, we expect that some sound will become trapped around that sound speed minimum in a sound channel.

In this sub-section, we will trace several rays from a submerged source to develop an understanding of the channel's propagation characteristics. Assume that there is a sound source at a depth of $800 \mathrm{~m}$, where the sound speed (by interpolation) is $1507 \mathrm{~m} / \mathrm{s}$. We start by calculating the upward angle from the source that will result in a horizontal ray at the top of the upper gradient, $z=560 \mathrm{~m}$, where the sound speed is $c_{\max }=1523 \mathrm{~m} / \mathrm{s}$. By Snell's law, as expressed in terms of the grazing angles in Eq. (11.38), $\theta_{1}=\cos ^{-1}(1507 / 1523)=0.145$; hence, $\theta_{1}=8.31^{\circ}$. The radius of the circular path of the ray, $\left|R_{l}\right|=c_{o} / g=\left(1523 \mathrm{~m} / \mathrm{s} \div 0.067 \mathrm{~s}^{-1}\right)=22.73 \mathrm{~km}$. Based on the diagram in Fig. 11.15, the sound ray intersects the top of the channel at a horizontal distance that is $r_{l}=\left|R_{l}\right| \sin \theta_{1}=3.3 \mathrm{~km}$ from the source.

From the top of the channel $(z=560 \mathrm{~m})$, the ray will return to the channel axis along the same circular path and will intersect the axis $(z=1112 \mathrm{~m})$ at $\theta_{2}=\cos ^{-1}(1486 / 1523)$, so $\theta_{2}=0.221 \mathrm{rad}=12.66^{\circ}$. The horizontal distance, $r_{2}-r_{1}=\left|R_{1}\right| \sin \theta_{2}=5.0 \mathrm{~km}$.

The ray enters the deep isothermal layer where $\left|R_{2}\right|=\left(1486 \mathrm{~m} / \mathrm{s} \div 0.0143 \mathrm{~s}^{-1}\right)=103.9 \mathrm{~km}$. (The center of that circle is only a geometrical "construction point," so the fact that it is located above the stratosphere should not be a cause for our concern.) Since we have defined the bottom of the channel as the location where the sound speed in the deep isothermal layer equals the sound speed at the top of the

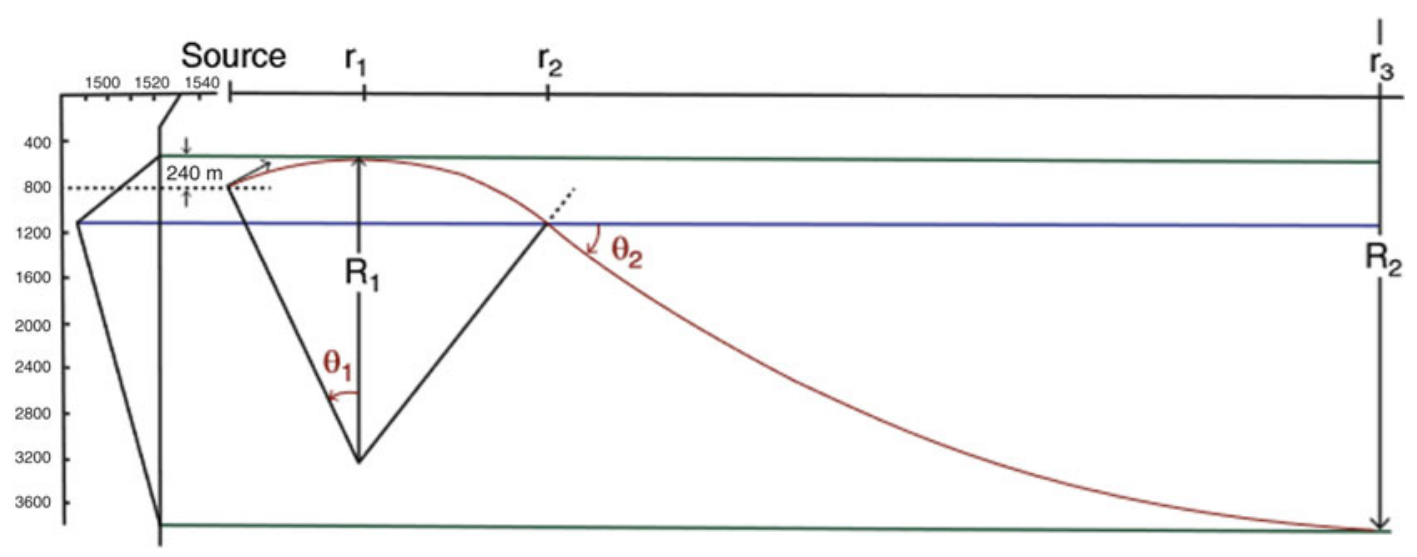

Fig. 11.15 The diagram of the sound speed vs. depth at the left is based on the piecewise-linear approximation of Fig. 11.11. There is a sound speed minimum at a depth of $z=1112 \mathrm{~m}$ that is the axis of a sound channel shown by the horizontal blue line. The velocity maxima are shown by the horizontal green lines at depths of $560 \mathrm{~m}$ and $3700 \mathrm{~m}$, corresponding to $c_{\max }=1523 \mathrm{~m} / \mathrm{s}$. The ray paths, ranges, depths, and especially the ray path radii, $\left|R_{l}\right|$ and $\left|R_{2}\right|$, are not drawn to scale. The limiting ray paths for a sound source located at a depth of $800 \mathrm{~m}$, and horizontal distance, $r=0$, are shown in red, launched at an angle of $\theta_{1}=8.31^{\circ}$ above the horizontal. The turning point for the ray in the upper layer occurs at a horizontal distance from the source of $r_{1}$, while the turning point for the ray in the lower layer occurs at a horizontal distance from the source of $r_{3}$. The ray crosses the channel axis at $r_{2}$ and enters the lower layer at an angle $\theta_{2}$ below the horizontal 


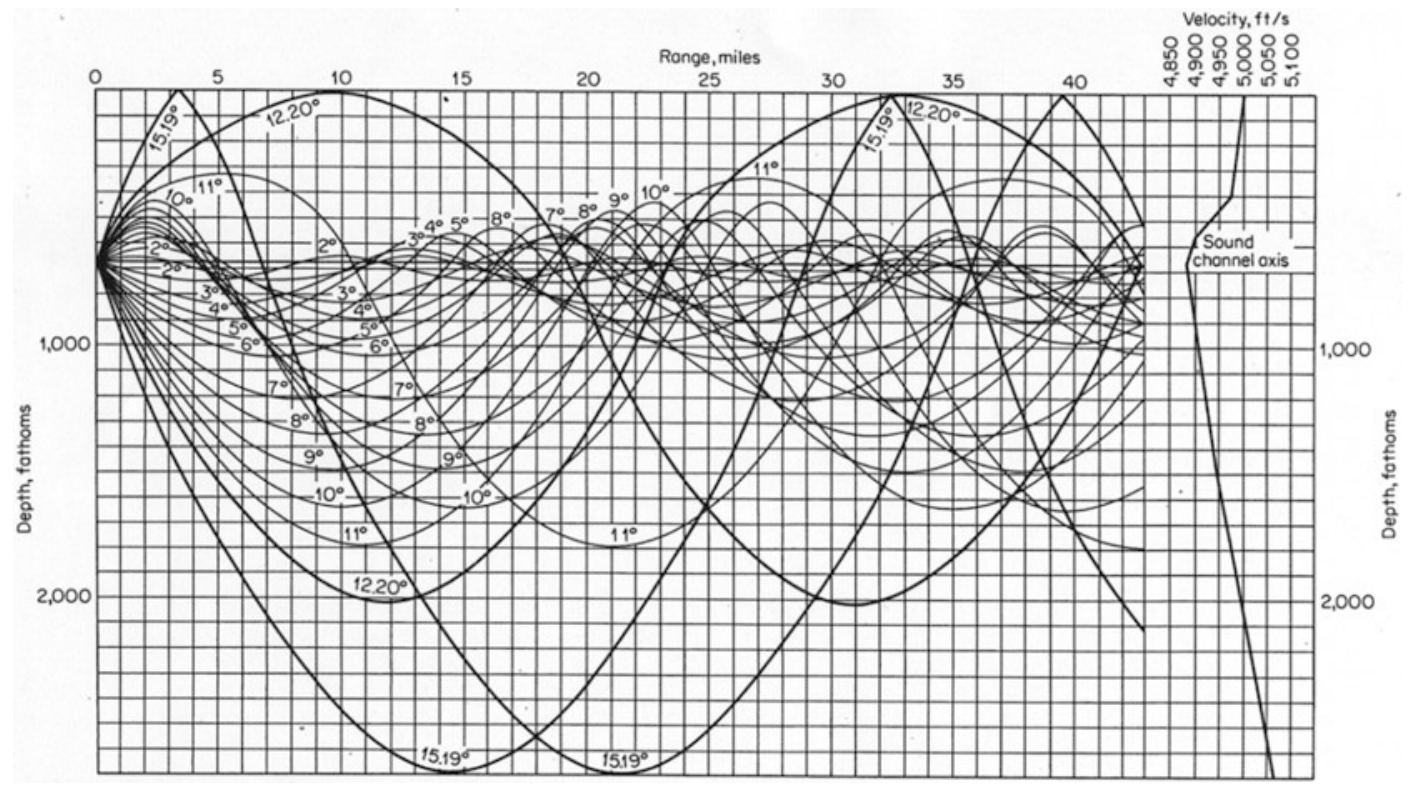

Fig. 11.16 Ray diagram for transmission paths in the deep sound channel for a source located on the channel axis. Depth is in fathoms $(1 \mathrm{fathom}=6 \mathrm{feet}=1.829 \mathrm{~m})$, range is in miles $(1 \mathrm{mi}=1.609 \mathrm{~km})$, and sound speed is in feet $/$ second $(1 \mathrm{fps}=30.48 \mathrm{~cm} / \mathrm{s})$, as a function of depth is provided at the right side of the graph. For angles $\theta>\theta_{\max }=12.2^{\circ}$, waves escaping the channel are reflected from the air-water interface [11]

main thermocline, $c_{\max }=1523 \mathrm{~m} / \mathrm{s}$, Snell's law will guarantee that it will reach that depth with a ray that is again horizontal, after traveling a horizontal distance, $r_{3}-r_{2}=\left|R_{2}\right| \sin \theta_{2}=22.8 \mathrm{~km}$. From that point at horizontal distance from the source, $r_{3}$, the path should repeat indefinitely with the cycle distance from the top of the channel back to the top of the channel being $2\left(r_{3}-\right.$ $\left.r_{l}\right)=55.6 \mathrm{~km}$.

To calculate the angular width of the sound radiated by our source located at $z=800 \mathrm{~m}$ below the surface, which will be trapped in the channel, we need to follow a ray that leaves the source at an angle below horizontal, $\theta_{-1}$, that will also take it to the depth $(z=3700 \mathrm{~m})$ where the sound speed is again $c_{\text {max }}$. That initially downward-directed ray will also have to cross the sound channel axis at $\theta_{2}=12.66^{\circ}$ if it is to become horizontal at $z=3700 \mathrm{~m}$. Again, Snell's law guarantees that $(1507 \mathrm{~m} / \mathrm{s} \div \cos$ $\left.\theta_{-1}\right)=\left(1486 \mathrm{~m} / \mathrm{s} \div \cos \theta_{2}\right)=1523 \mathrm{~m} / \mathrm{s}$, so $\left|\theta_{-1}\right|=\left|\theta_{1}\right|=8.31^{\circ}$. That result is easy to visualize since we can imagine a "virtual source" that is located $2 r_{1}$ away from the original source, where the ray makes an angle of $-8.31^{\circ}$ with respect to the horizontal. Any ray that leaves the source within $\pm 8.31^{\circ}$ will be trapped in the sound channel created by the two sound speed gradients.

Again, Snell's law makes it easy to see that an initially horizontal ray leaving our source at a depth of $z=800 \mathrm{~m}$ will go up and down in the channel from a depth of $z=800 \mathrm{~m}$ to a depth of $z=2581 \mathrm{~m}$, where the sound speed will again be $1507 \mathrm{~m} / \mathrm{s}$ and the rays will again be horizontal. Figure 11.16 diagrams ray paths from a more complicated sound channel for a source located on the channel axis [11].

The results illustrated in the previous example can be generalized by defining a maximum angle $\pm \theta_{\text {max }}$ (above or below the horizontal) that will result in the trapping of a wave launched at the channel axis, in terms of the minimum sound speed at the axis, $c_{\min }$, and the maximum sound speed, $c_{\max }$, at the bottom and top of the channel. 


$$
\cos \theta_{\max }=\frac{c_{\min }}{c_{\max }}
$$

Expanding $\cos \theta_{\max }$ for small angles and defining $\Delta c=c_{\max }-c_{\min }$, Eq. (11.39) can be approximated as $\theta_{\max } \cong \sqrt{2 D c / c_{\max }}$. When the source is above or below the height or depth of the channel by a distance, $z_{o}$, in a layer $D$ thick, Snell's law, and the requirement that a trapped ray at the channel boundary is horizontal, provides the range of trapping angles, $\theta_{ \pm 1}= \pm \theta_{\max } \sqrt{z_{o} / D}$, when the source is not located on the sound channel's axis. Along the channel axis, $z_{o}=D$. At the extremes, $z_{o}=0$, only the horizontal ray is trapped. The long-range transmission ability of the deep sound channel was used during World War II to rescue aviators that crashed at sea. ${ }^{9}$ A downed pilot would drop a small explosive charge that was rigged to detonate near the depth of the sound channel axis. If the sound were detected with two or more hydrophones, also located at the axis depth, the reception times could be used to localize the search by triangulation. Successful rescues were made this way using hydrophones connected to shore stations that were thousands of miles from the aircraft impacts. More recently, the same ability to make localizations at sea has been used for missile-impact location [12], and measurement of the time delays has been used to measure the mean temperature of the deep ocean [13].

\subsubsection{Propagation Delay*}

It is interesting to calculate the difference in the propagation times for sound traveling along different paths to reach the same horizontal distance from the source. Although the path along the axis is the shortest, it is also going through the medium with the minimum sound speed. The longer (curved) paths travel through water that has a faster sound speed. Does the shorter path beat the faster path?

Let's first consider the ray that is generated on the sound channel axis and travels along a circular path to the top of the channel. The initial angle of such a ray above the horizontal is $\theta_{\max } \cong$ $\sqrt{2 D c / c_{\max }}$, as already shown. The transit time, $T_{\text {upper }}$, for that ray can be calculated if we integrate from $\theta=0^{\circ}$ to $\theta=\theta_{\max }$, where the sound speed depends upon angle, $c(\theta)=c_{\max } \cos \theta$.

$$
T_{\text {upper }}=\int_{0}^{\theta_{\max }} \frac{|R| d \theta}{c(\theta)}=\int_{0}^{\theta_{\max }} \frac{|R| d \theta}{c_{\max } \cos \theta}=\frac{|R|}{c_{\max }} \int_{0}^{\theta_{\max }} \frac{d \theta}{\cos \theta}
$$

There is an analytical solution for the above definite integral, but simply writing the answer provides no useful physical insight.

$$
\int_{0}^{\theta_{\max }} \frac{d \theta}{\cos \theta}=\int_{0}^{\theta_{\max }} \sec \theta d \theta=\ln [\csc \theta-\cot \theta]_{0}^{\theta_{\max }}
$$

In these problems, the angles are generally small, so the series expansion of the sine and cosine functions and the binomial expansion can both be employed to simplify the integration and its interpretation.

$$
\int_{0}^{\theta_{\max }} \frac{d \theta}{\cos \theta} \cong \int_{0}^{\theta_{\max }} \frac{d \theta}{\left(1-\frac{\theta}{2}\right)} \cong \int_{0}^{\theta_{\max }}\left(1+\frac{\theta^{2}}{2}\right) d \theta=\theta_{\max }+\frac{\theta_{\max }^{3}}{6}
$$

The transit time for the axial ray, $T_{\text {axial }}$, that goes the same horizontal distance, $r=|R| \sin \theta_{\text {max }}$, is just $T_{\text {axial }}=r / c_{\text {min }}$. Since $c_{\text {min }}=c_{\max } \cos \theta_{\text {max }}$, the same approach can be used.

\footnotetext{
${ }^{9}$ In this context, the channel was known as the SOFAR channel, which stood for sound fixing and ranging.
} 


$$
T_{\text {axial }}=\frac{|R| \sin \theta_{\max }}{c_{\max } \cos \theta_{\max }} \cong \frac{|R|}{c_{\max }} \frac{\left(\theta_{\max }-\frac{\theta_{\max }}{6}\right)}{\left(1-\frac{\theta_{\max }^{2}}{2}\right)} \cong \frac{|R|}{c_{\max }}\left(\theta_{\max }-\frac{\theta_{\max }^{3}}{6}\right)\left(1+\frac{\theta_{\max }^{2}}{2}\right)
$$

We have to be vigilant at this point to be sure we are evaluating Eq. (11.43) to the same level of approximation as we had in Eq. (11.42), which is correct to third order in $\theta_{\max }$. The product of the two binomials includes a term that is linear in $\theta_{\max }$ as well as two terms that are third order.

$$
T_{\text {axial }}=\frac{|R|}{c_{\max }}\left(\theta_{\max }-\frac{\theta_{\max }^{3}}{6}+\frac{\theta_{\max }^{3}}{2}\right)=\frac{|R|}{c_{\max }}\left(\theta_{\max }+\frac{\theta_{\max }^{3}}{3}\right)
$$

These calculations demonstrate that the (longer) curved path is traversed in less time than the shorter (axial) path. We can use Eq. (11.44) with Eqs. (11.40) and (11.42) to approximate the travel time difference, $\Delta T$.

$$
\Delta T=T_{\text {axial }}-T_{\text {upper }} \cong \frac{|R|}{c_{\max }}\left[\left(\theta_{\max }+\frac{\theta_{\max }^{3}}{3}\right)-\left(\theta_{\max }+\frac{\theta_{\max }^{3}}{6}\right)\right]=\frac{|R|}{c_{\max }} \frac{\theta_{\max }^{3}}{6}
$$

\subsubsection{Under Ice Propagation}

Sound propagation under Arctic ice provides an interesting variation on the sound channel, since sound can be reflected specularly (i.e., $\theta_{r}=\theta_{i}$ ) from the ice sheet and the speed of sound under the ice increases monotonically with depth. The lack of solar heating at the surface causes the main thermocline, shown in Fig. 11.11, to be absent under Arctic ice. Typical ray paths under Arctic ice are shown in Fig. 11.17.

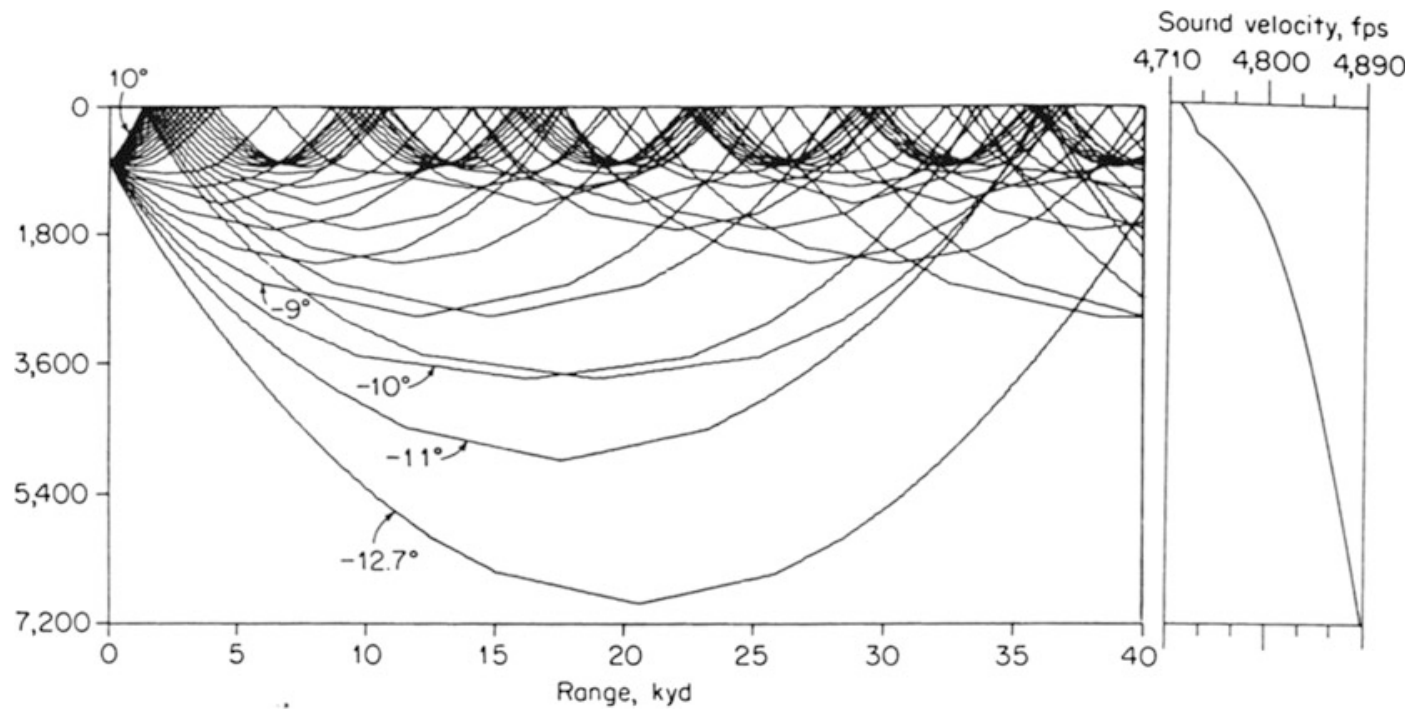

Fig. 11.17 Typical sound speed profile (right) and ray paths (left) under Arctic ice. Depth is in fathoms $(1$ fathom $=6$ feet $=1.829 \mathrm{~m})$, range is in kiloyards $(1 \mathrm{kyd}=0.9144 \mathrm{~km})$, and sound speed is in feet/second $(1 \mathrm{fps}=30.48 \mathrm{~cm} / \mathrm{s})[14]$ 


\subsubsection{Sound Focusing}

A final illustration of these refractive processes in constant sound speed gradients, at a much different scale than the global sound propagation in the deep sound channel, comes from the evolution of marine mammals. The Cuvier's beaked whale (Ziphius cavirostris) is a member of the Ziphiidae family of toothed whales. It relies on echolocation, but in an aqueous environment, the excessive hydrodynamic drag and turbulence noise that would be produced by external "ears" (i.e., pinna), like those found on land mammals, is unacceptable. In this whale, the "ear" is located internally behind the jaw (in green), as shown in Fig. 11.18.

Figure 11.19 demonstrates that this region acts like a lens. Parallel rays that enter the "channel" are focused to a single point (see Fig. 11.20). As we have done many times now, we can represent the distribution of sound speeds in the whale's mandible using a piecewise-linear approximation. In this
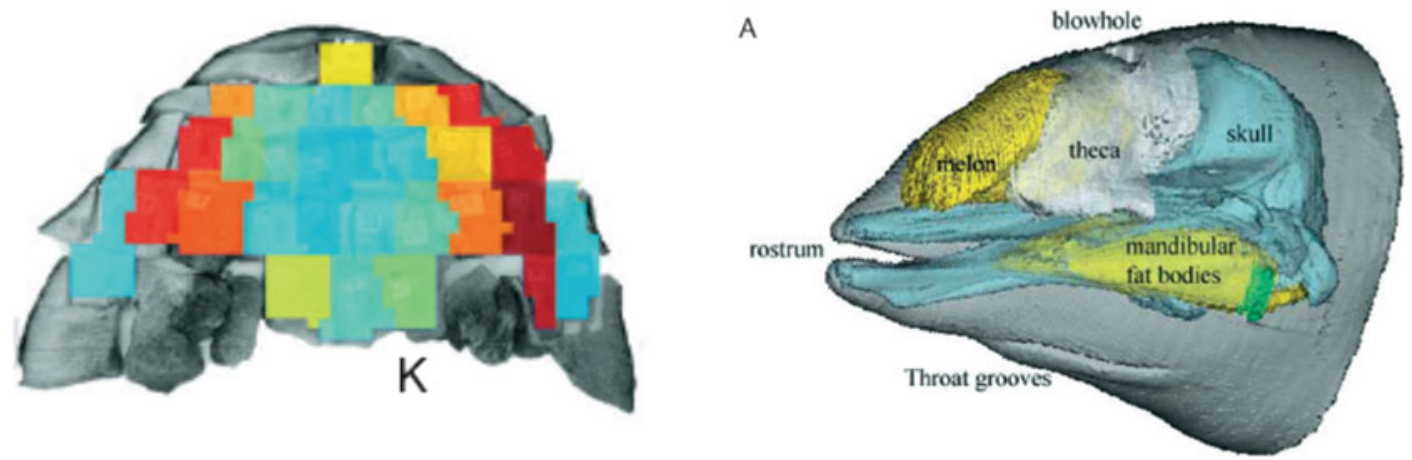

Fig. 11.18 The sound-sensing organ of the Cuvier's beaked whale is shown by the small green area in the drawing of the whale's head at the right. At the left is a tomographic slice through the mandibular fat body which acts as a sound channel (i.e., lens) to focus sounds from the water to the whale's sound-sensing organ. The sound speed of the mandibular fat body (in yellow at the right) shows the sound speed as a function of location (red corresponding to $1700 \mathrm{~m} / \mathrm{s}$ and light blue corresponding to $1300 \mathrm{~m} / \mathrm{s}$ ). Diagrams from Soldervilla, et al. [15]
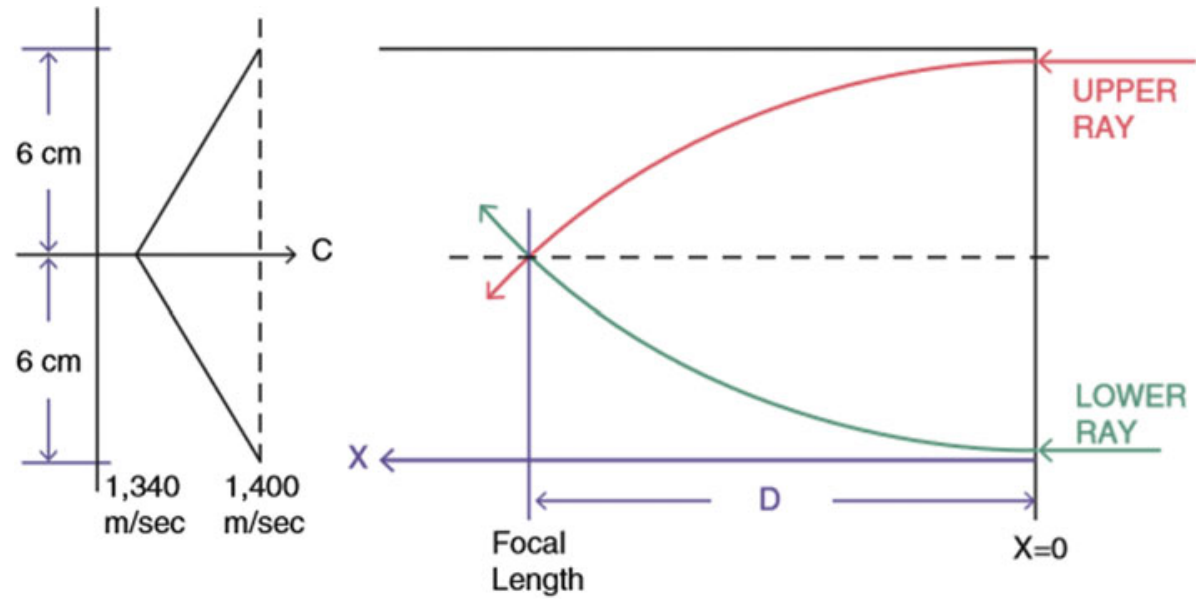

Fig. 11.19 A simplified approximation to the sound channel (i.e., lens) created by the mandibular fat body that assumes that the sound speed gradient is constant, with its minimum value $(1340 \mathrm{~m} / \mathrm{s})$ along the axis, and its maximum value $(1400 \mathrm{~m} / \mathrm{s})$ at the upper and lower extremes, $6.0 \mathrm{~cm}$ above and below the sound channel axis 

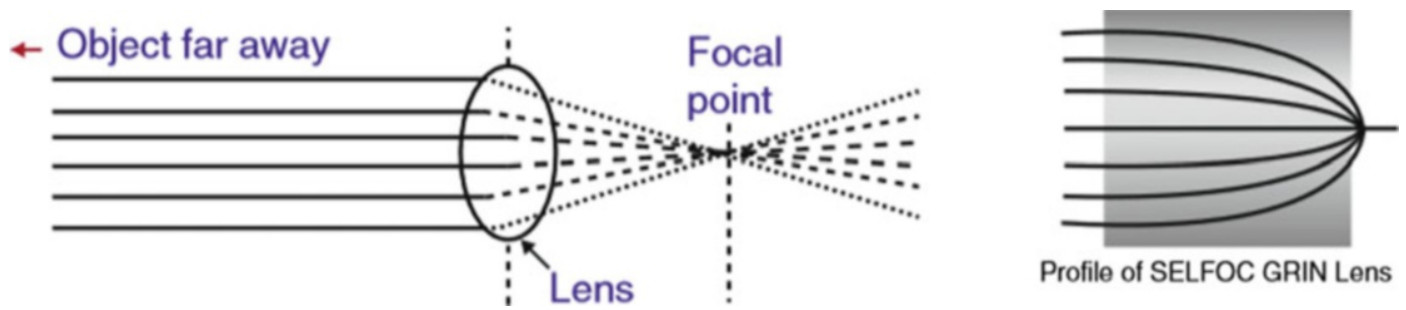

Fig. 11.20 (Left) Ray paths for an ordinary lens. (Right) Ray paths for a graded-index (GRIN) lens. In both cases, parallel rays enter the lens and are focused to a single point

case, the sound speed gradient $g=60 \mathrm{~m} / \mathrm{s} \div 0.06 \mathrm{~m}=1000 \mathrm{~s}^{-1}$; hence, $|R|=c_{o} / g=1.4 \mathrm{~m}$. Using Snell's law, $\cos \theta_{\max }=0.957$; hence, $\theta_{\max }=16.8^{\circ}$. This makes the "focal length" of the whale's acoustic lens, $d_{\text {focal }}=|R| \sin \theta_{\max }=40.5 \mathrm{~cm}$, just about the extent of the mandibular fat bodies.

The strategy of using a propagation speed profile to act as a lens is very popular in fiber-optic telecommunication systems and fiber-optic sensors. As shown in Fig. 11.20, this application is intended to capture light from an LED or solid-state laser and focus that light into the core of singlemode optical fibers that typically have waveguide (core) diameters on the order of 10 microns or less. In sensor applications [16-18], it usually is used to spread the light from an optical fiber over the surface of some sensing element after which a second GRIN lens injects the modulated light back into the optical fiber.

The graded-index lens was patented by Nippon Sheet Glass in 1968 and given the trademark SELFOC $^{\circledR}$. According to the accepted evolutionary timeline [19], marine mammals had produced the acoustical equivalent of GRIN lenses for at least 32 million years before the NSG patent was issued.

\section{Talk Like an Acoustician}

$\begin{array}{ll}\text { Cavitation effects } & \text { Fermat's principle } \\ \text { Pressure reflection coefficient } & \text { Critical angle } \\ \text { Pressure transmission coefficient } & \text { Total internal reflection } \\ \text { Pressure release boundary } & \text { Angle of intromission } \\ \text { Impedance matching layer } & \text { Refraction } \\ \text { Antireflective coating } & \text { Piecewise-linear approximation } \\ \text { Trace wavelength } & \text { Ray tracing } \\ \text { Specific acoustic impedance } & \text { Grazing angle } \\ \text { Specular reflection } & \text { Sound channel } \\ \text { Diffuse reflection } & \text { SOFAR channel } \\ \text { Snell's law } & \end{array}$

\section{Exercises}

1. Air-water interface. A $1.0 \mathrm{kHz}$ planewave in water with pressure amplitude, $|\widehat{\mathbf{p}}|=100 \mathrm{~Pa}$, is normally incident on the air-water interface (i.e., the propagation direction is at a right angle to the air-water surface; hence, $\theta_{i}=0^{\circ}$ ). You may assume the speed of sound in water is $1500 \mathrm{~m} / \mathrm{s}$ and the speed of sound in air is $343 \mathrm{~m} / \mathrm{s}$. The density of water can be taken as $1000 \mathrm{~kg} / \mathrm{m}^{3}$ and of air to be $1.19 \mathrm{~kg} / \mathrm{m}^{3}$.

(a) Transmitted amplitude. What is the amplitude of the pressure that is transmitted into the air?

(b) Trace velocity. What is the "trace velocity" of the waves along the interface? 
(c) Intensities. What is the intensity of the incident wave in the water and the transmitted wave in air?

(d) Transmission loss. Express the reduction in the intensity of the wave when it crosses the interface from the water into the air in decibels.

(e) Oblique incidence. If the angle of incidence is increased to $45^{\circ}$, what is the amplitude of the pressure transmitted into the air?

(f) Trace velocity. What is the "trace velocity" of the waves along the interface?

(g) Transmitted pressure. If the planewave originates in that air and makes an angle of $45^{\circ}$ to the normal, what is the magnitude of the acoustic pressure of the sound in the water?

2. Matching layer. What is the density and sound speed required for a $1.0-\mathrm{cm}$-thick layer of material that produces complete transmission of sound from water into steel if the frequency of the sound wave is $20 \mathrm{kHz}$ ? Let $\rho_{\text {water }}=1000 \mathrm{~kg} / \mathrm{m}^{3}$ and $c_{\text {water }}=1500 \mathrm{~m} / \mathrm{s}, \rho_{F e}=7700 \mathrm{~kg} / \mathrm{m}^{3}$, and $c_{F e}=6100 \mathrm{~m} / \mathrm{s}$.

3. Alcohol on water. An acoustics experiment is performed in an aquarium partially filled with water $\left(c=1500 \mathrm{~m} / \mathrm{s}\right.$ and $\left.\rho_{m}=1000 \mathrm{~kg} / \mathrm{m}^{3}\right)$. At the bottom of the aquarium, an ultrasonic transducer shoots a narrow beam of sound up to the surface of the water at an angle of $45^{\circ}$, and the sound beam reflects back down to the bottom of the aquarium where it hits a distance along the bottom that is $16.0 \mathrm{~cm}$ from the transducer. This situation is diagrammed in the upper portion of Fig. 11.21. You may assume the bottom of the aquarium is perfectly absorbing, so the beam does not bounce back up again and that the problem is entirely two dimensional.

After making the required measurement, the experimenter comes back from lunch and finds that a layer of ethyl alcohol $\left(c=1150 \mathrm{~m} / \mathrm{s}\right.$ and $\left.\rho_{m}=790 \mathrm{~kg} / \mathrm{m}^{3}\right)$ has carefully been poured on top of the water in the aquarium. (Good grief!) Now a second beam of sound comes down and hits the bottom at a distance of $22.0 \mathrm{~cm}$ from the ultrasonic transducer, as diagrammed in the lower portion of Fig. 11.21. How thick is the layer of alcohol on top of the water, assuming that the two liquids do not mix?

4. Oblique incidence at a water-sediment boundary. A sinusoidal planewave with effective pressure $p_{\text {rms }}=100 \mathrm{~Pa}$ is incident at $45^{\circ}$ on a silt bottom with $\rho_{\text {silt }}=2000 \mathrm{~kg} / \mathrm{m}^{3}$ and $c_{\text {silt }}=2000 \mathrm{~m} / \mathrm{s}$.

(a) Angle of refraction. What angle does the planewave make with respect to the normal once it enters the silt bottom?

(b) Transmitted pressure. What is the effective pressure of the wave in the silt bottom?

(c) Angle of intromission. At what angle does the planewave have to approach the silt from the water so that there is $100 \%$ transmission into the silt and no wave reflected back into the water?

Fig. 11.21 (Above) The sound beam of an ultrasonic transducer bounces of the free surface of a water-filled aquarium. (Below) the beam is displaced after a layer of alcohol is added to the surface
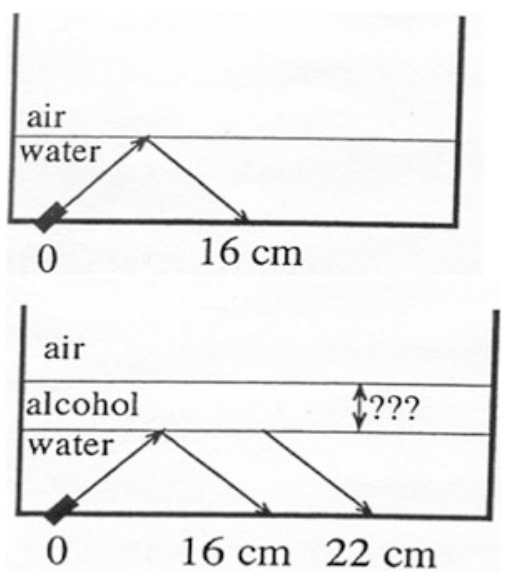
5. Critical ray and skip distance in a "mixed layer." The sound speed at the water's surface is $1490 \mathrm{~m} / \mathrm{s}$ and increases linearly to $c_{o}=1491.5 \mathrm{~m} / \mathrm{s}$ with depth to the bottom of the mixed layer at $D=100 \mathrm{~m}$. Below that depth, there is a constant negative sound speed gradient $g_{2}=-0.045 \mathrm{~s}^{-1}$. For an underwater sound source (projector) at a depth, $z_{s}=60.0 \mathrm{~m}$, determine the critical (grazing) angle, $\theta_{o}$, for the limiting ray that remains trapped in the mixed layer and the "skip distance," $r_{s}$, between the successive locations where the critical ray intersects the surface.

6. Arctic refraction. The water in the Arctic is nearly isothermal. Sound speed increases with depth because of the pressure effect. Assume the surface temperature is $0{ }^{\circ} \mathrm{C}$, the salinity is $35 \mathrm{ppt}$, and the sound speed, in meters/second, as a function of depth, is given by the Eq. (11.46), where $z$ is the depth below the surface expressed in meters [20].

$$
c(z)=1449+0.016 z
$$

The turning depth of the ray is $2.0 \mathrm{~km}$ where the sound speed is $c(2 \mathrm{~km})=1481 \mathrm{~m} / \mathrm{s}$.

(a) Initial grazing angle. What is the initial angle that ray makes with the water-ice interface? Report the angle which the ray makes with the water's surface, not the angle with respect to the normal to the surface.

(b) Return to the surface. What is the distance along the surface at which the ray returns to the ice-water interface?

\section{References}

1. E.A. Neppiras, Acoustic cavitation. Phys. Rep. 61(3), 159-251 (1980)

2. V.O. Knudsen, C.M. Harris, Acoustical Design in Architecture (Acoustical Society of America, 1978). See Rigid Partitions, pp. 208-210; ISBN 0-88318-267-X.

3. R.B. Lindsay, Mechanical Radiation (McGraw-Hill, 1960). See $\$ 9.6$

4. T.F.W. Embleton, Mufflers, in Noise and Vibration Control, ed. by L. L. Beranek, (McGraw-Hill, 1971), p. 379

5. R.P. Feynman, R.B. Leighton, M. Sands, The Feynman Lectures on Physics, vol I (Addison-Wesley, 1965). Chapter 26-3

6. C.W. Holland, Geoacoustic inversion for fine-grained sediments. J. Acoust. Soc. Am. 111(4), 1560-1564 (2002)

7. T.B. Gabrielson, Refraction of sound in the atmosphere. Acoustics Today 2(2), 7-17 (2006)

8. C.C. Leroy, Simple equations for accurate and more realistic calculation of the speed of sound in sea water. J. Acoust. Soc. Am. 46(1)(Pt. 2), 216-226 (1969)

9. L. M. Jones \& W. A. VonWinkle, Sound Velocity Profiles in an Area South of Bermuda, Underwater Sound Laboratory Report No. 632 (1965)

10. C.D. Ross, Civil War Acoustic Shadows (White Mane Books, Shippensburg, 2001). "Outdoor sound propagation in the US Civil War," Appl. Acoustics 59, 137-147 (2000)

11. M. Ewing, J.L. Worzel, Long-range sound transmission, in Propagation of Sound in the Ocean, vol. 3, Geo. Soc. Am. Memo. 27, (1948)

12. H.H. Baker, Missile impact localization system. Bell Telephone Lab. Res. 39, 195 (1961). A similar on-line discussion is available at https://insidegnss.com/on-target-offshore-scoring-of-precision-guided-munitions/

13. K.G. Sabra, B. Cornuelle, W.A. Kuperman, Sensing deep-ocean temperatures. Phys. Today 69(2), 32-38 (2016)

14. R. J. Urick, Sound Propagation in the Sea (Defense Advanced Research Projects Agency Report 1979). Reprinted as Principles of Underwater Sound (McGraw-Hill, 1975); ISBN 0-07-066086-7

15. M.S. Soldervilla et al., Cuvier's beaked whale (Ziphius cavirostris) head tissue physical properties and CT imaging. J. Exp. Biol. 208, 2319-2332 (2005)

16. S.L. Garrett, M.R. Brininstool, J.T. Newmaster, T. Hofler, Fiber optic angular orientation sensor using digital serial encoding. US Pat. No. 5,042,157 (Aug. 27, 1991) 
17. A. Migliori, G.W. Swift, S.L. Garrett, Remotely readable fiber optic compass. US Pat. No. 4,577,414 (Mar. 25, 1986)

18. M.R. Brininstool, J.T. Newmaster, S.L. Garrett, Fiber-optic remote angular position sensor including a polarization track. US Pat. No. 5,073,711 (Dec. 17, 1991)

19. A. Berta, J.L. Sumich, Marine Mammals: Evolutionary Biology (Academic, 1999); ISBN 0-12-093225-3.

20. C.S. Clay, H. Medwin, Acoustical Oceanography (Wiley-Interscience, 1977), pp. 90-91; ISBN 0-471-16041-5.

Open Access This chapter is licensed under the terms of the Creative Commons Attribution 4.0 International License (http://creativecommons.org/licenses/by/4.0/), which permits use, sharing, adaptation, distribution and reproduction in any medium or format, as long as you give appropriate credit to the original author(s) and the source, provide a link to the Creative Commons license and indicate if changes were made.

The images or other third party material in this chapter are included in the chapter's Creative Commons license, unless indicated otherwise in a credit line to the material. If material is not included in the chapter's Creative Commons license and your intended use is not permitted by statutory regulation or exceeds the permitted use, you will need to obtain permission directly from the copyright holder. 